\title{
The Role of SUMO-1 in Cardiac Oxidative Stress and Hypertrophy
}

\author{
Ahyoung Lee, ${ }^{1, *}$ Dongtak Jeong,, ${ }^{1, \star}$ Shinichi Mitsuyama, Jae Gyun Oh, Lifan Liang,, Yoshiyuki Ikeda, ${ }^{2}$ \\ Junichi Sadoshima, ${ }^{2}$ Roger J. Hajjar, and Changwon Kho ${ }^{1}$
}

\begin{abstract}
Aims: Small ubiquitin-like modifier type 1 (SUMO-1) has been shown to play a critical role in the dysfunction of the cardiac isoform of sarcoplasmic reticulum calcium ATPase (SERCA2a) pump in the setting of heart failure. In cardiac hypertrophy, the role of SUMO-1 has not been defined and our study's goals were to examine the effects of modulating SUMO-1 on the hypertrophic response both in vitro and in vivo and to examine whether oxidative stress (during cardiac hypertrophy) is abrogated by SUMO-1 gene transfer. Results: In mice undergoing transverse aortic constriction (TAC), SUMO-1 levels increased slightly during the compensated stage of hypertrophy and then dropped sharply during the transition to heart failure. In isolated cardiomyocytes, SUMO-1 gene transfer inhibited the hypertrophic response in the presence of phenylephrine. Adeno-associated vector type 9 (AAV9) gene transfer of SUMO-1 prevented the heart from undergoing hypertrophy after TAC and prevented the development of left ventricular dysfunction. Furthermore, SUMO-1 gene transfer blocked the negative effects of $\mathrm{H}_{2} \mathrm{O}_{2}$ on SERCA2a activity in cardiac myocytes, while in vivo indices of oxidative stress were decreased by SUMO-1 in cardiac hypertrophy and heart failure. Innovation and Conclusion: The results of this study indicate that post-translational modifications of SERCA2a caused by the toxic environment of the hypertrophied and failing myocardium can be prevented by SUMO-1. Antioxid. Redox Signal. 21, 1986-2001.
\end{abstract}

\section{Introduction}

$\mathbf{C}$ ARDIAC HYPERTROPHY induces perturbations in signaling pathways and initiates a program of molecular, biochemical, and structural modifications that lead to a remodeled ventricle $(14,25,64)$. Cardiac hypertrophy is initially an adaptive response (48), but sustained cardiac hypertrophy results in an increased risk of sudden death and progression to heart failure (33). Recently, experimental and clinical studies have shown that cardiovascular redoxsensitive signaling pathways are closely linked to the onset of cardiac hypertrophy and heart failure. Many of the molecular mechanisms of cardiac hypertrophy are involved in redox-regulated pathways such as cardiomyocyte excitationcontraction coupling, differentiation, hypertrophy, and stress responses (6). In fact, in the hypertrophied and failing hearts, elevated levels of reactive oxygen species (ROS) and cardiomyocyte oxidative stress are associated with maladaptive ventricular remodeling and a progressive de- cline in cardiovascular function $(13,22,27,30)$. Increased indices of oxidant stress have been measured in patients with congestive heart failure. In clinical studies, patients with heart failure were found to have evidence of lipid

\section{Innovation}

Novel findings of this study include that the demonstration of (i) small ubiquitin-like modifier type 1 (SUMO-1) expression blocks hypertrophic responses in isolated cardiomyocytes stimulated by phenylephrine; (ii) SUMO-1 gene transfer abrogates the hypertrophic response to pressure overload in vivo; and (iii) SUMO-1 expression protects SERCA2a from oxidative stress. These observations suggest that post-translational modifications of SERCA2a caused by the toxic environment of the hypertrophied and failing myocardium can be prevented by the expression of SUMO-1.

\footnotetext{
${ }^{1}$ Department of Cardiology, Cardiovascular Research Center, Icahn School of Medicine at Mount Sinai, New York, New York.

${ }^{2}$ Department of Cell Biology \& Molecular Medicine, Rutgers New Jersey Medical School, Newark, New Jersey.

*These authors contributed equally to this work.
} 
peroxidation and elevated 8-iso-prostaglandin $\mathrm{F}_{2 \alpha}$ levels $(17,44,45,62)$; whereas in experimental models of hypertrophy, investigators have been able to directly measure increased ROS production from cardiomyocytes $(27,36)$. These findings have been corroborated in studies that measured ROS levels in explanted human hearts at the time of cardiac transplantation (56). Furthermore, a number of neurohormonal and mechanical stressors that are associated with the heart failure phenotype augment ROS generation $(2,54,66)$.

Impaired cardiac isoform of sarcoplasmic reticulum calcium ATPase (SERCA2a) function underlies many of the effects of pressure overload-induced hypertrophy on cardiomyocyte performance, and it has been shown to be critical in the progression of compensatory hypertrophy to heart failure $(28,49,51)$. Our group along with other investigators has shown that increasing the expression of SERCA2a by gene transfer in various animal models of heart failure improves cardiac function. Following a long line of investigation, SERCA2a gene therapy is now undergoing testing in human clinical trials $(19,29,70)$. SERCA2a protein is also sensitive to oxidative stress. Hydrogen peroxide and hydroxyl radicals directly inhibit ATPase activity by interfering with ATP binding, thereby impairing SR calcium pump rate (67). Furthermore, oxidative modifications of SERCA2 have also been identified. Increased tyrosine nitration of SERCA2a is positively correlated with aging (68). In addition, cystein oxidation of SERCA2 contributes to the decrease in its activity that leads to impaired calcium handling and relaxation in the aging heart (55).

We have recently found that the levels and activity of SERCA2a in cardiomyocytes are modulated in parallel with the levels of a cytoplasmic protein, small ubiquitin-like modifier type 1 (SUMO-1). We also found that SERCA2a is SUMOylated at lysine residues 480 and 585 and that this post-translational modification is responsible for stabilizing SERCA2a as well as for enhancing its activity. Furthermore, we showed that increasing SUMO-1 levels led to restoration of SERCA2a levels, improved hemodynamic performance, and reduced mortality in a murine model of heart failure (34). More recently, we demonstrated that SUMO-1 gene transfer and its combination with SERCA2a led to a reversal of heart failure in a porcine model of ischemia-induced heart failure (63).

It is well known that global increases in protein SUMOylation occur in response to cellular stress. Recent studies have also shown an intimate relationship between SUMO and oxidative stress. Oxidative stress can affect the conjugation profile of SUMO to a target protein in a concentrationand time-dependent fashion $(5,47)$. In human neuronal cells, overexpression of SUMO increases cell survival in the presence of oxygen/glucose deprivation, suggesting that SUMO is a component of ischemic tolerance (40). However, it is unknown what happens to the expression of SUMO-1 in the setting of compensated hypertrophy and the transition from hypertrophy to heart failure and whether modulating SUMO-1 in this setting alters the phenotype. In this study, we examined the effects of overexpressing SUMO-1 on the hypertrophic response both in vitro and in vivo. Since oxidative stress is a critical component of cardiac hypertrophy and heart failure, the effects of SUMO-1 gene transfer on oxidative stress are also examined.

\section{Results}

SUMO-1 is differentially expressed throughout the progress of heart failure

To gain insights into a possible role of SUMOylation in the stress response during the progression to heart failure, SUMO-1 expression levels were quantified in transverse aortic constriction (TAC) mice at different time points and related to cardiac function. Western blot analysis showed that protein levels of both SUMO-1 and SERCA2a were altered by TAC-induced pressure overload (Fig. 1). Both SUMO-1 and SERCA2a protein levels were increased in mice at 2 weeks of TAC (23\% in SUMO- 1 level and $12 \%$ in SERCA2a level, respectively, vs. no TAC control), when brain natriuretic peptide (BNP) level was significantly increased (1.9-fold, vs. no TAC control) and systolic function was significantly increased during compensatory hypertrophy (fractional shortening [FS]; $75.9 \%$ in 2 weeks TAC vs. $63.3 \%$ in baseline), and declined thereafter. At 12 weeks of TAC when LV function was severely decreased (FS; $39 \%$ vs. $63.3 \%$ in baseline, $p<0.05)$, SUMO-1 and SERCA2a protein expression was decreased (32\% decreased in SUMO-1 level and $48 \%$ decreased in SERCA2a level compared with baseline). TAC also altered SUMOylated SERCA2a levels in a time-dependent manner. SUMOylation status of SERCA2a was increased at 2 weeks of TAC but greatly decreased at 12 weeks of TAC. These data suggested that loss of SUMO-1 levels contributes to the decreased SUMOylation of SERCA2a during the transition to heart failure.

\section{SUMO-1 overexpression inhibits the phenylephrine-induced hypertrophic response}

First, the ability of SUMO-1 to inhibit cardiac hypertrophy was evaluated in vitro. The cultured neonatal rat cardiomyocytes were infected with either Ad.SUMO-1 or Ad.SERCA2a or Ad. $\beta$-gal. At $24 \mathrm{~h}$ after infection, the cardiomyocytes were further stimulated with $100 \mu M$ phenylephrine (PE), an agonist for cardiac hypertrophy, for $24 \mathrm{~h}$. PE-treated cardiomyocytes showed a significantly $(p<0.05)$ increased in mRNA level of fetal genes such as ANF (2-fold, vs. no PE), BNP (1.6-fold, $v s$. no PE), and $m y h 7$ (3.1-fold, $v s$. no PE) in the Ad. $\beta$-gal-infected cardiomyocytes. In contrast, no significant PE-induced increases in these typical markers for cardiac hypertrophy were observed with either Ad.SUMO-1- or Ad.SERCA2a-infected cardiomyocytes (Fig. 2A). Another feature of the hypertrophic response of cardiomyocytes is a pronounced increase in protein synthesis. The increased protein synthesis induced by PE treatment (2.9-fold, vs. no PE), as determined by ${ }^{3} \mathrm{H}$-leucine incorporation, was significantly $(p<0.05)$ suppressed by SUMO-1 overexpression (0.54-fold, vs. Ad. $\beta$-gal control) and SERCA2a overexpression (0.45-fold, vs. Ad. $\beta$-gal control) (Fig. 2B). Stimulation with PE results in an increase in cell size of cardiomyocytes infected with Ad. $\beta$-gal. In contrast, no significant increase in cell size was observed in cardiomyocytes infected with either Ad.SUMO-1 or Ad.SERCA2a after $\mathrm{PE}$ treatment (Fig. 2C). Interestingly, PE treatment caused a $57 \%$ decrease in SERCA2a protein expression, whereas SERCA2a protein level was maintained in SUMO-1 overexpressing cells compared with Ad. $\beta$-gal overexpressing cells (Supplementary Fig. S1; Supplementary Data are available online at www.liebertpub.com/ars). At the molecular level, 


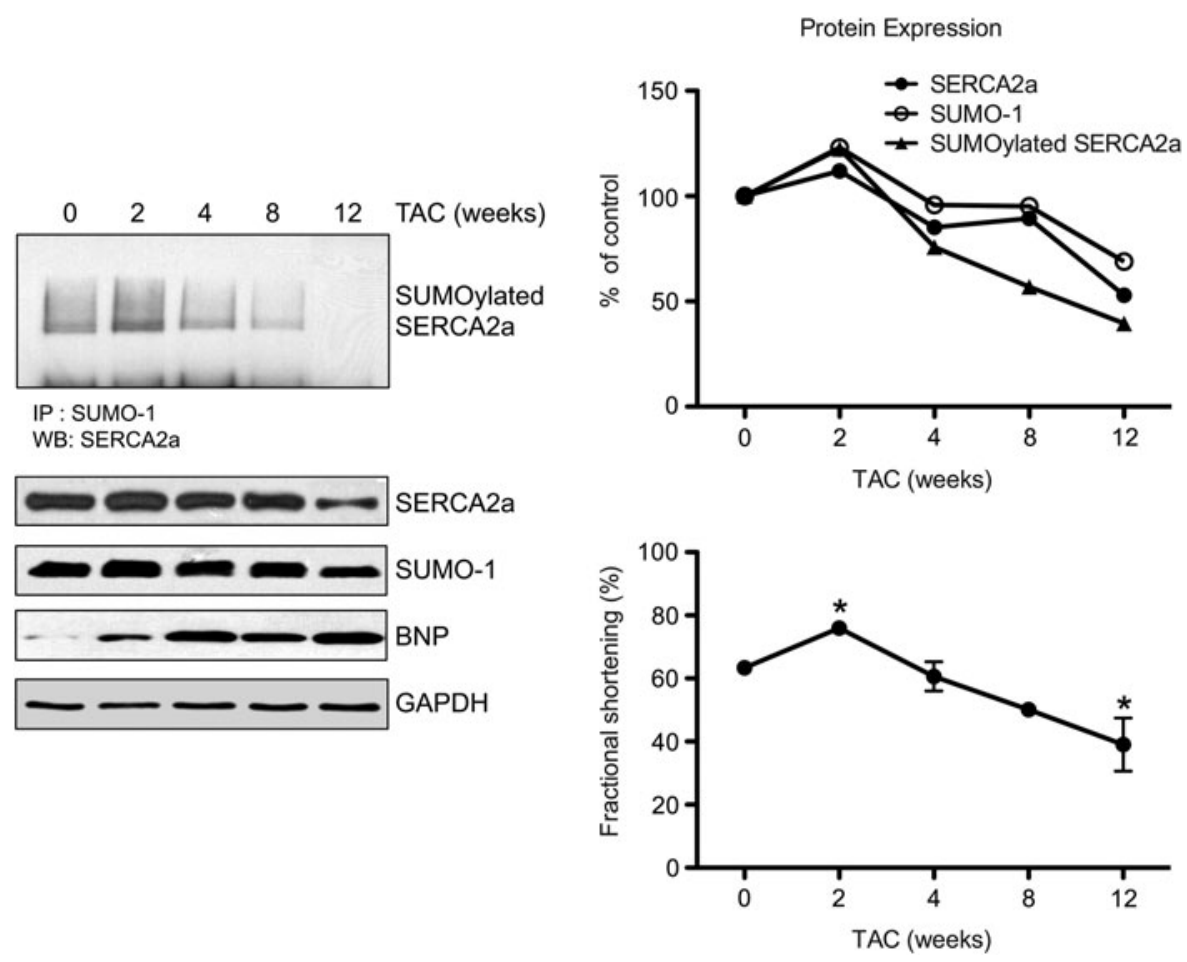

FIG. 1. Changes of SUMO-1 in transition from compensated cardiac hypertrophy to heart failure in a mouse model. Western blot analysis of SUMO-1 protein levels in the TAC-operated mouse hearts. Time is shown as weeks after TAC operation. The mice were characterized by degree of cardiac hypertrophy and heart failure, which was measured by changes in cardiac BNP and SERCA2a protein levels (top) and left ventricular FS, an index of cardiac systolic contractile function (bottom). SUMO-1, SERCA2a, and BNP levels are quantified by ImageJ software. GAPDH was used as a loading control to normalize the data. ${ }^{*} p<0.05$ versus before TAC (0 week) using Student's $t$-test. $n=3$ per each group. Endogenous SERCA2a SUMOylation was determined by IP with anti-SUMO-1 followed by and WB with anti-SERCA2a antibody. The experiments shown are representative of three independent Western blots with similar results. BNP, brain natriuretic peptide; FS, fractional shortening; GAPDH, glyceraldehyde 3-phosphate dehydrogenase; IP, immunoprecipitation; SERCA2a, cardiac isoform of sarcoplasmic reticulum calcium ATPase; SUMO-1, small ubiquitin-like modifier type 1; TAC, transverse aortic constriction; WB, Western blotting.

activation of mitogen-activated protein kinase (MAPK) has been associated with the development of cardiac hypertrophy. To investigate the effect of SUMO-1 on MAPK signaling, cardiomyocytes were treated with $100 \mu M \mathrm{PE}$ for $24 \mathrm{~h}$ and total cell lysates were analyzed by Western blotting for phosphorylation of three major MAPKs. As shown in Figure 2D, PE increased phosphorylation of extracellular signal-regulated kinase (ERK) (1.2-fold, vs. no PE), p38 MAPK (1.6-fold, vs. no PE), c-Jun N-terminal kinase (JNK) (2.5-fold, vs. no PE), and c-Jun (3-fold, vs. no PE), downstream target for the JNK and the major substrates of stress-responsive MAPKs. However, adenoviral overexpression of SUMO-1 was sufficient to prevent PE-induced activation of all three kinases ( $p-E R K$ : 0.63-fold, p-p38: 0.57-fold, and p-JNK: 0.65-fold respectively, $v s$. Ad. $\beta$-gal control, $p \leq 0.05)$ and c-Jun $(0.41$-fold, $v s$. Ad. $\beta$ gal control, $p=0.02)$. Interestingly, SUMO-1 overexpression suppressed MAPK pathways to the same degree as SERCA2a overexpression (p-ERK: 0.7-fold and p-JNK: 0.5-fold respectively, vs. Ad. $\beta$-gal control $p \leq 0.05$ ). There was no significant difference between the cardiomyocytes infected with either Ad.SUMO- 1 or Ad.SERCA2a and Ad. $\beta$-gal in the absence of PE treatment (Supplementary Fig. S2). Taken together, these results indicated that the overexpression of SUMO-1 inhibited agonist-induced cardiac hypertrophy in neonatal rat cardiomyocytes. SUMO-1 overexpression may counteract hypertrophic stimulation by inhibiting MAPK pathways.

FIG. 2. SUMO-1 overexpression inhibits PE-induced hypertrophic response in cardiomyocytes. (A) The neonatal cardiomyocytes were infected with the indicated adenoviruses and further stimulated with PE (100 $\mu M)$ for $24 \mathrm{~h}$. The relative expression levels of hypertrophic association genes such as ANF, BNP, and myh7 were determined by semiquantitative RT-PCR $(n=3)$. (B) The protein synthesis rates after PE stimulation were evaluated by measuring ${ }^{3} \mathrm{H}-\mathrm{leucine}$ incorporation. Data indicate the mean value \pm SD of three independent experiments. (C) Cell size measurement. (D) MAPK activities after the stimulation of neonatal cardiomyocytes with PE. Cardiomyocytes were infected with indicated adenoviruses for $24 \mathrm{~h}$ and then treated with PE $(100 \mu M)$ for $24 \mathrm{~h}$. Cell extracts were examined for amounts of phosphorylated ERK, p38, JNK, and c-Jun using phospho-specific antibodies. Total protein levels of ERK, p38, JNK, and c-Jun were also analyzed. The relative intensity was quantitated using ImageJ software. Data indicate the mean \pm SD of three independent experiments. ${ }^{*} p<0.05$ versus Ad. $\beta$-gal; ${ }^{*} p<0.05$ versus Ad. $\beta$-gal + PE. ERK, extracellular signal-regulated kinase; JNK, c-Jun N-terminal kinase; MAPK, mitogen-activated protein kinase; PE, phenylephrine. 
A

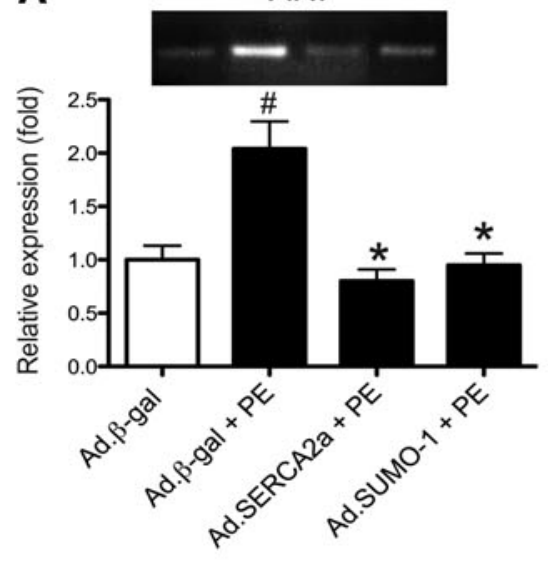

B 응

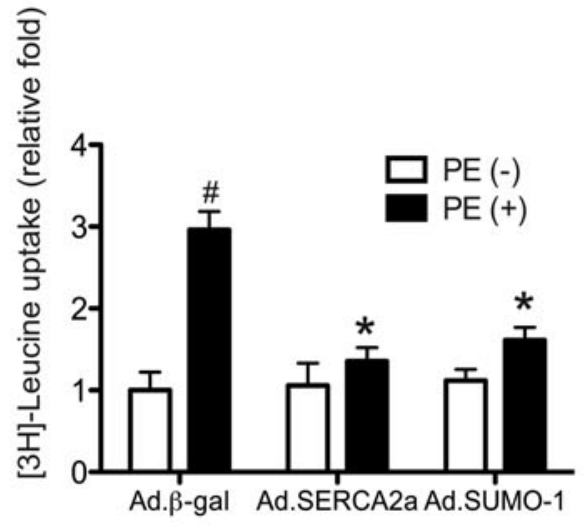

$B N P$

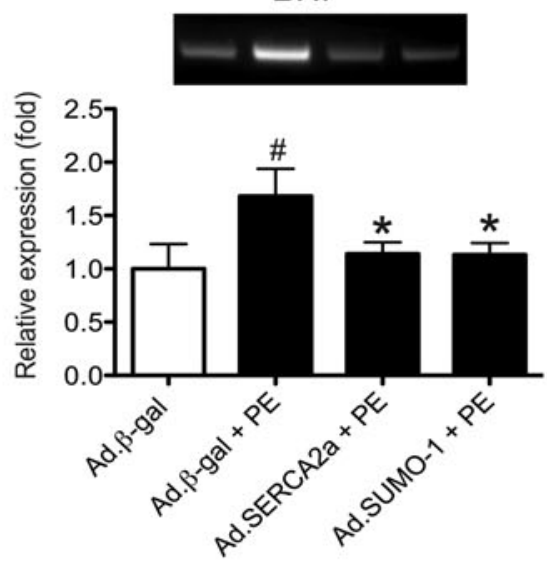

myh7

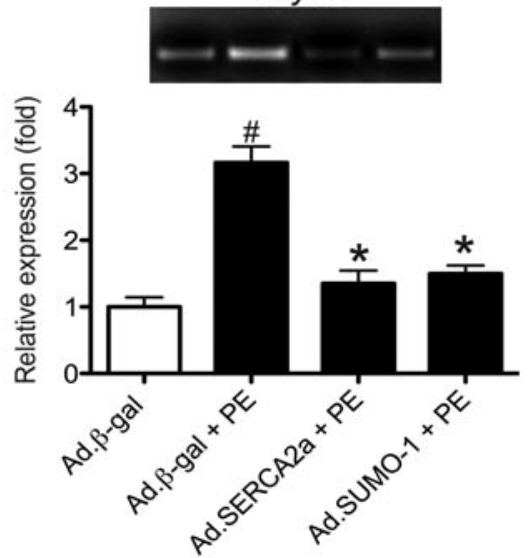

D

Ad. Ad. Ad. Ad.

$\beta$-gal $\beta$-gal SERCA2aSUMO-1

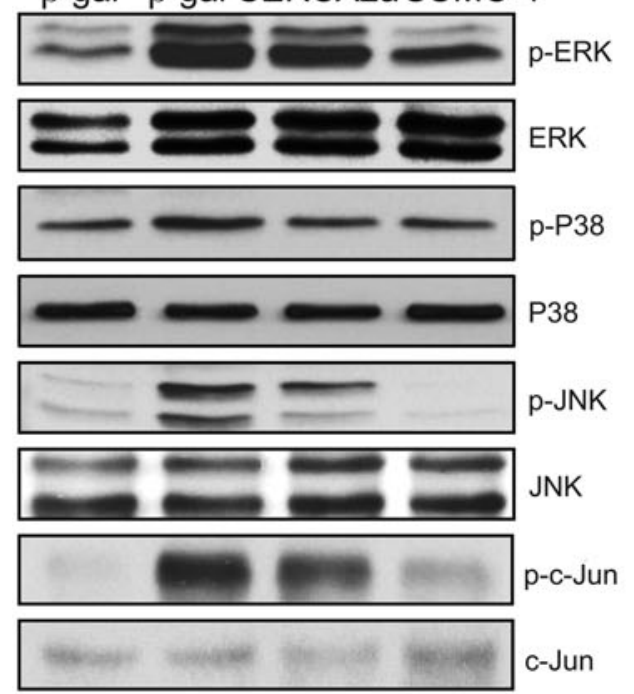

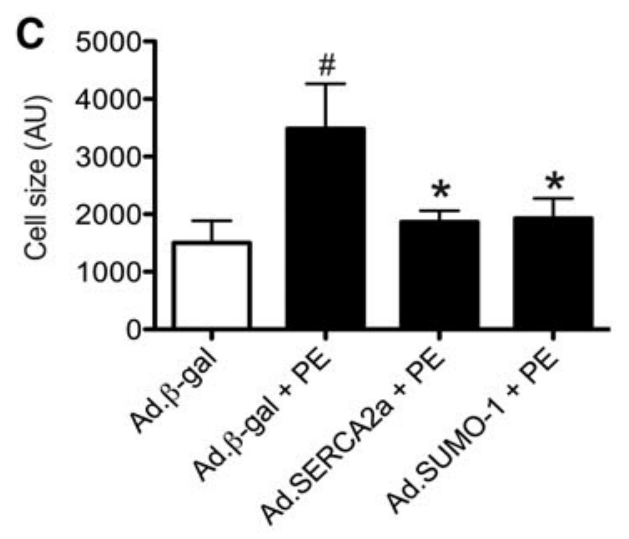

pERKJERK

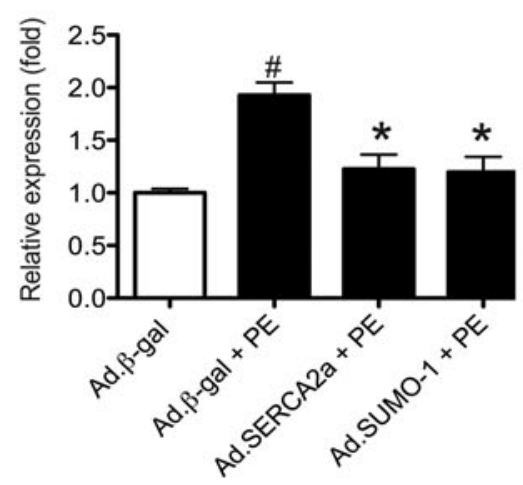

pJNK/JNK

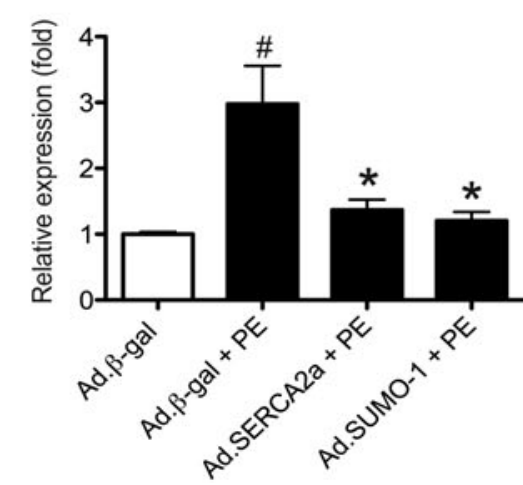

pP38/P38

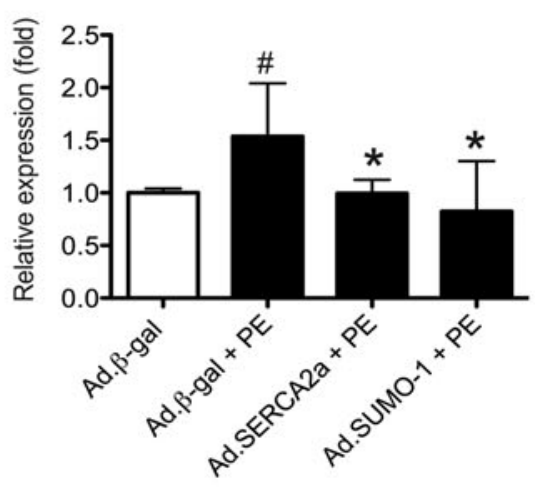

p-cJun/Jun

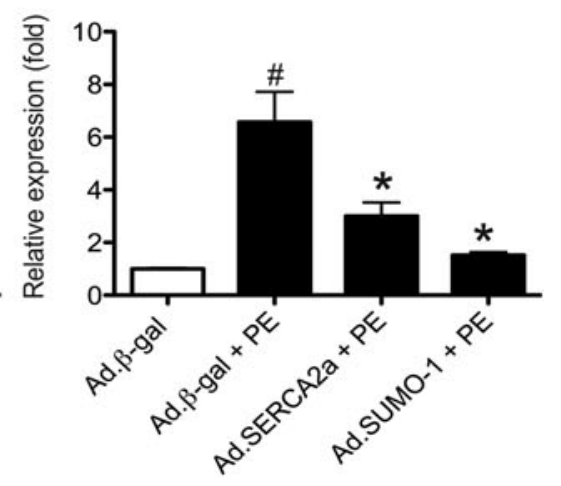




\section{A}

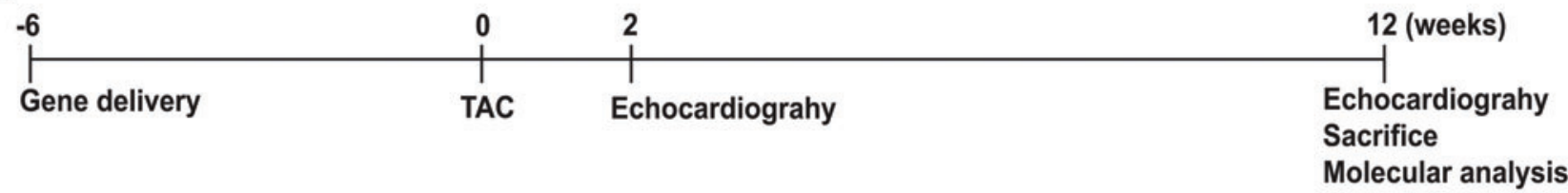

B

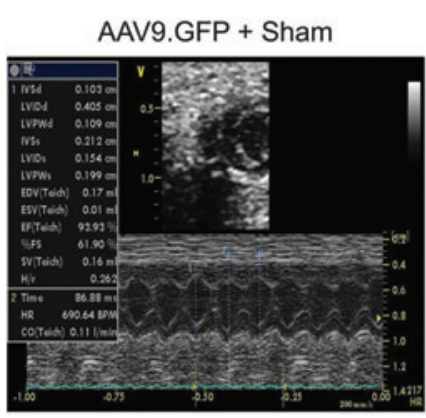

2 weeks TAC

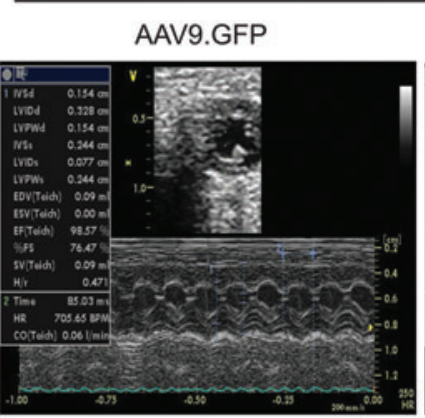

AAV9.SERCA2a

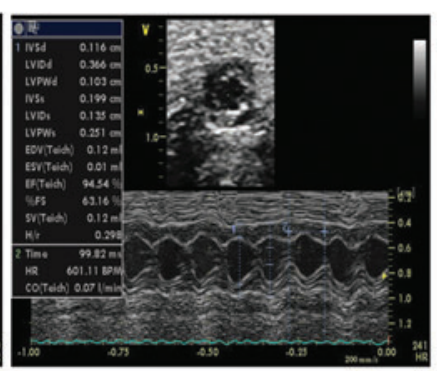

AAV9.SUMO-1

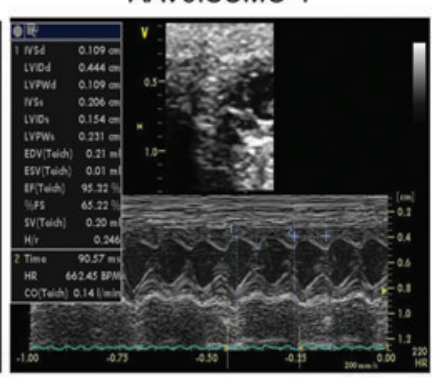

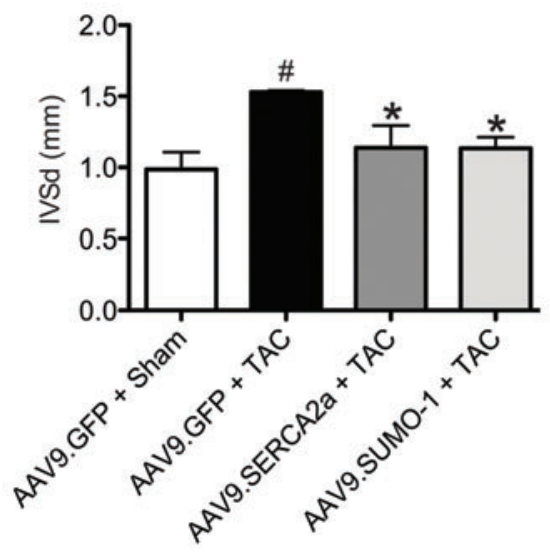
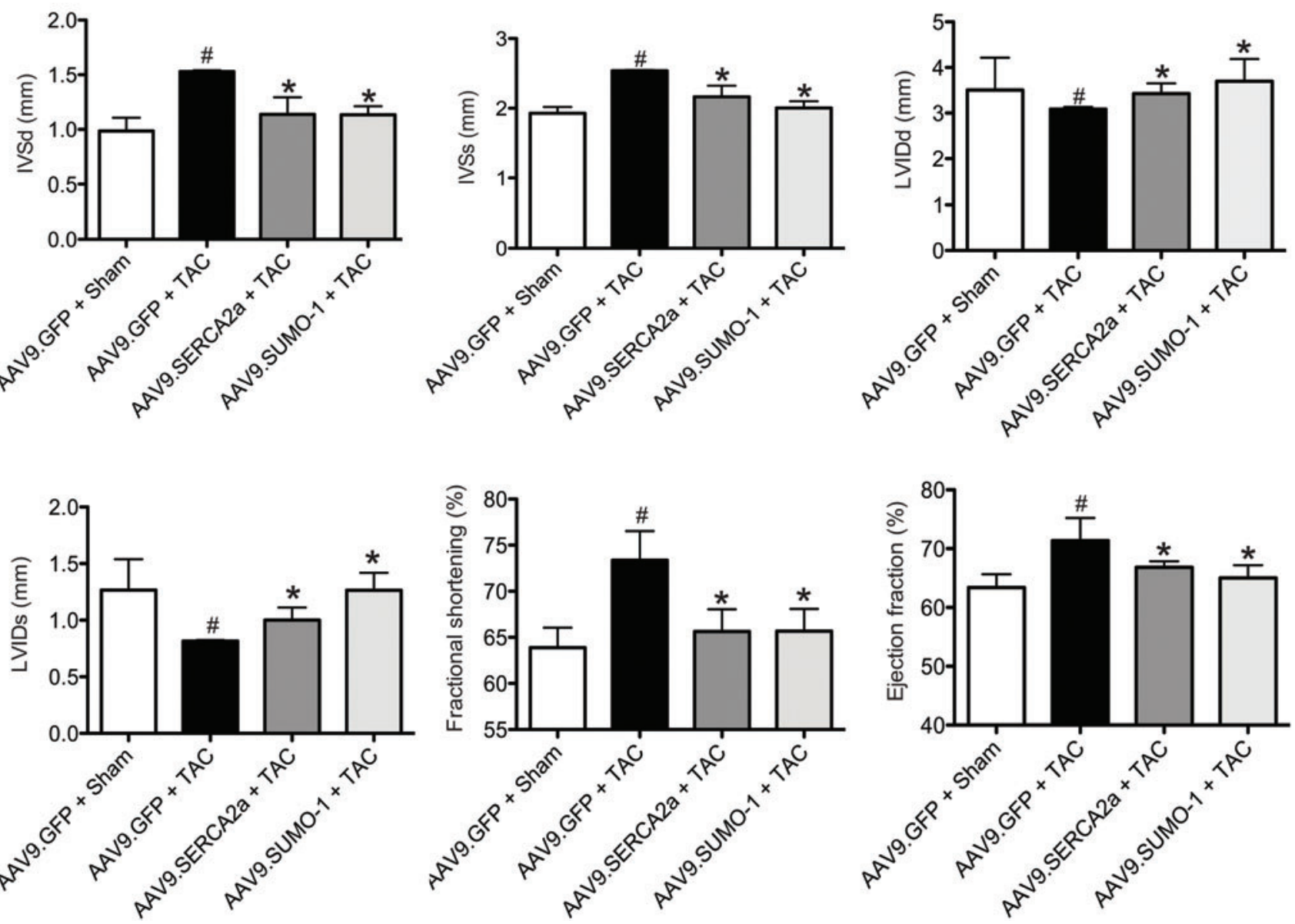

FIG. 3. SUMO-1 overexpression preserves cardiac hypertrophy at 2 weeks after TAC mice. (A) Study design. Sixweek-old male B6C3/F1 mice received AAV9 vectors carrying SUMO-1, SERCA2a, and GFP via tail-vein injection. After 6 weeks of gene delivery, cardiac function was measured by echocardiography and then, the mice were subjected to either TAC or sham operation. Cardiac function was assessed before surgery (baseline) and at 2 weeks and 12 weeks postsurgery followed by tissue collection. (B) Transthoracic echocardiography was performed using a Vivid 7000 equipped (GE Healthcare, Salt Lake City, UT) with an H13L transducer. Representative transthoracic M-mode echocardiogram for AAV9.GFP+sham, AAV9.GFP + TAC, AAV9.SERCA2a + TAC, and AAV9.SUMO-1 + TAC mice at 2 weeks of surgery is shown (top). Several echocardiographic parameters (bottom) were measured, such as IVSd, IVSs, LVPWd, LVIDd, LVIDs, FS, and EF. ${ }^{p} p<0.05$ versus GFP control with sham operation; * $p<0.05$ versus GFP control at 2 weeks of TAC. AAV9, adeno-associated vector type 9; EF, ejection fraction; GFP, green fluorescent protein; IVSd, interventricular septal thickness in diastole; IVSs, interventricular septal thickness in systole; LVIDd, left ventricular internal diameter in diastole; LVIDs, left ventricular internal diameter in systole. To see this illustration in color, the reader is referred to the web version of this article at www.liebertpub.com/ars 
Table 1. Echocardiographic Parameters at 6 Weeks After Tail Vein Injection WITH AAV9.GFP, AAV9.SERCA2A, OR AAV9.SUMO-1

\begin{tabular}{lccc}
\hline & AAV9.GFP $(\mathrm{n}=5)$ & AAV9.SERCA2 $a(\mathrm{n}=5)$ & AAV9.SUMO-1 $(\mathrm{n}=5)$ \\
\hline IVSd (mm) & $1.00 \pm 0.15$ & $0.95 \pm 0.05$ & $1.00 \pm 0.08$ \\
LVIDd (mm) & $3.47 \pm 0.39$ & $3.47 \pm 0.23$ & $3.16 \pm 0.54$ \\
LVPWd (mm) & $1.00 \pm 0.05$ & $1.08 \pm 0.14$ & $0.95 \pm 0.06$ \\
IVSs (mm) & $1.94 \pm 0.21$ & $1.87 \pm 0.05$ & $1.91 \pm 0.09$ \\
LVIDs (mm) & $1.27 \pm 0.17$ & $1.23 \pm 0.10$ & $1.17 \pm 0.20$ \\
LVPWs (mm) & $1.91 \pm 0.17$ & $1.85 \pm 0.16$ & $1.81 \pm 0.11$ \\
EF (\%) & $94.50 \pm 1.27$ & $95.09 \pm 0.58$ & $94.47 \pm 0.60$ \\
FS (\%) & $63.21 \pm 3.08$ & $64.45 \pm 1.42$ & $62.90 \pm 1.34$ \\
HR (bpm) & $637.41 \pm 50.77$ & $629.94 \pm 50.25$ & $630.84 \pm 73.27$ \\
\hline
\end{tabular}

All data represent the mean value \pm the SD of cardiac functional parameters.

$\mathrm{EF}$, ejection fraction; FS, fractional shortening; HR, heart rate; IVSd, interventricular septal thickness in diastole; IVSs, interventricular septal thickness in systole; LVIDd, left ventricular internal diameter in diastole; LVIDs, left ventricular internal diameter in systole; LVPWd, left ventricular posterior wall thickness in diastole; LVPWs, left ventricular posterior wall thickness in systole.

\section{SUMO-1 pretreatment prevents pressure} overload-induced cardiac hypertrophy

To determine whether SUMO-1 overexpression inhibits hypertrophic response in vivo, SUMO-1 was pretreated by adeno-associated vector type 9 (AAV9)-mediated gene delivery. AAV9 is known to be a cardiotropic vector. AAV9 .SUMO-1 was injected into B6C3/F1 male mice through the tail vein at a dose of $5 \times 10^{10}$ viral genomes per mouse (vg/ mice). Mice injected with same doses of either AAV9 .SERCA2a or AAV9.GFP viruses were used as positive and negative control groups, respectively. Six weeks after the injection, mice were subjected to TAC operation. Cardiac function was accessed by echocardiography at day 0 (baseline), 2, and 12 weeks after TAC operation (Fig. 3A).

There were no significant differences in LV function and LV dimension between the gene-delivered groups before TAC (Table 1). By contrast, at 2 weeks of TAC, AAV9.GFP preinjected mice exhibited hypertrophic phenotypes with significant increases in interventricular septal thickness in diastole (IVSd; $p<0.05, v s$. sham), interventricular septal thickness in systole (IVSs; $p<0.05, v s$. sham), left ven- tricular posterior wall thickness in diastole (LVPWd; $p<0.05, v s$. sham), left ventricular posterior wall thickness in systole (LVPWs, $p<0.05 v s$. sham), and LV FS ( $p<0.05$, vs. sham) and significant decreases in left ventricular internal diameter in diastole (LVIDd; $p<0.05, v s$. sham) and left ventricular internal diameter in systole (LVIDs; $p<$ 0.05 , vs. sham). SUMO-1 pretreatment, however, significantly suppressed these hypertrophic phenotypes. The increase in LV end-diastolic and systolic wall thickness and ventricular posterior wall thickness in diastole caused by TAC was significantly preserved in AAV9.SUMO-1 preinjected mice compared with AAV9.GFP preinjected control $(p<0.05)$. Consistently, LV FS and ejection fraction (EF) was maintained in SUMO-1 pretreated mice (FS; $65.58 \%$ in 2 weeks' TAC, $v s .64 .23 \%$ in sham and EF; $95.52 \%$ in TAC, vs. 95\% in 2 weeks' sham) (Fig. 3B and Table 2). Similar results were obtained in the AAV9.SERCA2a preinjected group. TAC-induced hypertrophy was strongly prevented by the pretreatment with SERCA2a. These data indicated that SUMO-1 pretreatment abrogated the development of left ventricular hypertrophy induced by pressure overload in vivo.

Table 2. Echocardiographic Parameters from Mice Injected with AAV9.GFP, AAV9.SERCA2A, or AAV9.SUMO-1 at 2 Weeks After Sham or Transverse Aortic Constriction Surgery

\begin{tabular}{|c|c|c|c|c|c|c|}
\hline & \multicolumn{3}{|c|}{ Sham } & \multicolumn{3}{|c|}{$T A C$} \\
\hline & $\begin{array}{c}A A V 9 . G F P \\
(\mathrm{n}=5)\end{array}$ & $\begin{array}{c}A A V 9 . S E R C A 2 a \\
(\mathrm{n}=5)\end{array}$ & $\begin{array}{c}\text { AAV9.SUMO-1 } \\
(\mathrm{n}=5)\end{array}$ & $\begin{array}{c}A A V 9 . G F P \\
(\mathrm{n}=6)\end{array}$ & $\begin{array}{c}A A V 9 . S E R C A 2 a \\
(\mathrm{n}=6)\end{array}$ & $\begin{array}{c}\text { AAV9.SUMO-1 } \\
(\mathrm{n}=6)\end{array}$ \\
\hline IVSd (mm) & $0.96 \pm 0.07$ & $1.01 \pm 0.06$ & $1.04 \pm 0.06$ & $1.50 \pm 0.08^{\mathrm{a}}$ & $1.13 \pm 0.15^{\mathrm{b}}$ & $1.13 \pm 0.07^{\mathrm{b}}$ \\
\hline LVIDd (mm) & $3.72 \pm 0.36$ & $3.44 \pm 0.47$ & $3.29 \pm 0.64$ & $2.88 \pm 0.31^{\mathrm{a}}$ & $3.42 \pm 0.22^{\mathrm{b}}$ & $3.69 \pm 0.48^{\mathrm{b}}$ \\
\hline LVPWd (mm) & $1.03 \pm 0.06$ & $1.02 \pm 0.04$ & $1.01 \pm 0.07$ & $1.46 \pm 0.15^{\mathrm{a}}$ & $1.11 \pm 0.12^{\mathrm{b}}$ & $1.11 \pm 0.11^{\mathrm{b}}$ \\
\hline IVSs (mm) & $1.93 \pm 0.09$ & $1.93 \pm 0.07$ & $2.06 \pm 0.23$ & $2.54 \pm 0.01^{\mathrm{a}}$ & $2.16 \pm 0.16^{\mathrm{a}, \mathrm{b}}$ & $2.11 \pm 0.10^{\mathrm{b}}$ \\
\hline LVIDs (mm) & $1.36 \pm 0.18$ & $1.34 \pm 0.21$ & $1.18 \pm 0.22$ & $0.81 \pm 0.07^{\mathrm{a}}$ & $1.17 \pm 0.11^{\mathrm{b}}$ & $1.26 \pm 0.15^{\mathrm{b}}$ \\
\hline LVPWs (mm) & $1.75 \pm 0.19$ & $1.77 \pm 0.17$ & $1.70 \pm 0.14$ & $2.10 \pm 0.20^{\mathrm{a}}$ & $2.04 \pm 0.23^{\mathrm{a}}$ & $2.01 \pm 0.21^{\mathrm{a}}$ \\
\hline $\mathrm{EF}(\%)$ & $94.64 \pm 1.07$ & $94.67 \pm 1.17$ & $95.00 \pm 0.81$ & $97.59 \pm 0.43^{\mathrm{a}}$ & $95.52 \pm 0.99^{\mathrm{b}}$ & $95.52 \pm 0.91^{\mathrm{b}}$ \\
\hline $\mathrm{FS}(\%)$ & $63.53 \pm 2.52$ & $63.54 \pm 2.70$ & $64.23 \pm 2.01$ & $71.97 \pm 1.86^{\mathrm{a}}$ & $65.62 \pm 2.41^{\mathrm{b}}$ & $65.58 \pm 2.41^{\mathrm{b}}$ \\
\hline HR (bpm) & $653.82 \pm 33.46$ & $615.81 \pm 27.76$ & $606.03 \pm 45.73$ & $608.57 \pm 53.97$ & $651.54 \pm 44.58$ & $634.58 \pm 40.93$ \\
\hline
\end{tabular}

All data represent the mean value \pm the $\mathrm{SD}$ of cardiac functional parameters.

${ }_{1}^{a} p<0.05$ compared with the sham groups.

${ }^{\mathrm{b}} p<0.05$ compared with the AAV9.GFP control; significant effect of TAC.

TAC, transverse aortic constriction. 
Table 3. Echocardiographic Parameters from Mice Injected with AAV9.GFP, AAV9.SERCA2A, Or AAV9.SUMO-1 at 12 Weeks After Sham or Transverse Aortic Constriction Surgery

\begin{tabular}{|c|c|c|c|c|c|c|}
\hline & \multicolumn{3}{|c|}{ Sham } & \multicolumn{3}{|c|}{$T A C$} \\
\hline & $\begin{array}{c}A A V 9 . G F P \\
(\mathrm{n}=6)\end{array}$ & $\begin{array}{c}\text { AAV 9.SERCA } 2 a \\
(\mathrm{n}=6)\end{array}$ & $\begin{array}{c}A A V 9 . S U M O-1 \\
(\mathrm{n}=6)\end{array}$ & $\begin{array}{c}A A V 9 . G F P \\
(\mathrm{n}=6)\end{array}$ & $\begin{array}{c}A A V 9 . S E R C A 2 a \\
(\mathrm{n}=6)\end{array}$ & $\begin{array}{c}A A V 9 . S U M O-1 \\
(\mathrm{n}=6)\end{array}$ \\
\hline IVSd (mm) & $0.99 \pm 0.09$ & $1.04 \pm 0.10$ & 1.03 & $1.01 \pm 0.20^{\mathrm{a}}$ & $1.22 \pm 0.13^{\mathrm{b}}$ & 1.07 \\
\hline LVIDd (mm) & $3.57 \pm 0.21$ & $3.66 \pm 0.37$ & $3.18 \pm 0.42$ & $4.40 \pm 0.67^{\mathrm{a}, \mathrm{b}, \mathrm{c}}$ & $3.66 \pm 0.39^{c}$ & $3.67 \pm 0.20^{b, c}$ \\
\hline LVPWd (mm) & $0.99 \pm 0.06$ & $1.01 \pm 0.05$ & $1.03 \pm 0.09$ & $1.03 \pm 0.18^{\mathrm{a}}$ & $1.30 \pm 0.17^{\mathrm{a}, \mathrm{b}, \mathrm{c}}$ & $1.12 \pm 0.13$ \\
\hline IVSs $(\mathrm{mm})$ & $1.83 \pm 0.18$ & $2.00 \pm 0.21$ & $1.93 \pm 0.09$ & $1.85 \pm 0.41^{\mathrm{a}}$ & $2.11 \pm 0.17$ & $2.02 \pm 0.09^{c}$ \\
\hline LVIDs (mm) & $1.46 \pm 0.38$ & $1.22 \pm 0.27$ & $1.18 \pm 0.23$ & $2.43 \pm 0.95^{\mathrm{a}, \mathrm{c}}$ & $1.70 \pm 0.37^{\mathrm{a}, \mathrm{b}}$ & $1.55 \pm 0.19^{\mathrm{a}, \mathrm{b}, \mathrm{c}}$ \\
\hline LVPWs (mm) & $1.98 \pm 0.32$ & $1.78 \pm 0.12$ & $1.82 \pm 0.08$ & $1.93 \pm 0.29^{\mathrm{a}, \mathrm{b}}$ & $1.92 \pm 0.19$ & $1.86 \pm 0.10^{\mathrm{b}}$ \\
\hline $\mathrm{EF}(\%)$ & $94.11 \pm 1.53$ & $94.81 \pm 1.16$ & $94.48 \pm 1.36$ & $76.54 \pm 9.78^{\mathrm{a}, \mathrm{b}, \mathrm{c}}$ & $89.08 \pm 3.49^{\mathrm{a}, \mathrm{b}, \mathrm{c}}$ & $90.33 \pm 2.27^{\mathrm{a}, \mathrm{b}, \mathrm{c}}$ \\
\hline $\mathrm{FS}(\%)$ & $63.18 \pm 2.57$ & $63.86 \pm 2.75$ & $63.10 \pm 3.20$ & $40.42 \pm 8.30^{\mathrm{a}, \mathrm{b}, \mathrm{c}}$ & $53.95 \pm 5.91^{\mathrm{a}, \mathrm{b}, \mathrm{c}}$ & $55.33 \pm 4.08^{\mathrm{a}, \mathrm{b}, \mathrm{c}}$ \\
\hline $\mathrm{HR}$ (bpm) & $637.87 \pm 39.12$ & $624.92 \pm 58.00$ & $675.55 \pm 36.65$ & $589.34 \pm 46.27$ & $637.24 \pm 47.94$ & $663.13 \pm 54.56$ \\
\hline
\end{tabular}

All data represent the mean value \pm the SD of cardiac functional parameters.

${ }^{a} p<0.05$ compared with the 2 weeks' TAC.

${ }^{\mathrm{b}} p<0.05$, compared with the AAV9.GFP control; Significant effect of TAC.

${ }^{c} p<0.05$ compared with the sham groups.

\section{SUMO-1 pretreatment prevents development of heart failure}

The beneficial effects of SUMO-1 pretreatment were also observed in the transition from hypertrophy to failure. All the remaining viable mice were subjected to echocardiography to assess the progression of heart failure. As expected, the prolonged pressure overload induced the typical phenotypes of heart failure. Increased LV wall thickness was significantly reduced at 12 weeks of TAC $(p<0.05, v s .2$ weeks of TAC) (Table 3). The degree of LV dilation assessed as LV end-diastolic and systolic diameters was significantly increased in AAV9.GFP preinjected mice at 12 weeks of TAC $(p<0.05$, vs. 2 weeks of TAC and $p<0.05$, vs. sham). Systolic function was significantly decreased in AAV9.GFP preinjected mice as demonstrated by decreases of LV FS and EF after TAC, as compared with the sham $(p<0.05)$ and 2 weeks of TAC $(p<0.05)$ (Fig. 4A and Table 3). However, development of heart failure was significantly ameliorated in AAV9.SUMO-1 preinjected mice compared with the AAV9 .GFP preinjected control group $(p<0.05)$. The LV chamber cavity was significantly maintained in the AAV9.SUMO-1 group $(p<0.05)$. LV FS and EF were significantly enhanced in AAV9.SUMO-1 pretreated mice compared with the green fluorescent protein (GFP) preinjected control animals $(p<0.05)$ (Fig. 4A and Table 3). At 12 weeks of TAC, the heart weight-tobody weight ratio was significantly higher in the AAV9.GFP preinjected group (ratio 9.25 \pm 2.0 ) than both AAV9.SUMO-1 (ratio 5.29 \pm 0.67 ) and AAV9.SERCA2a (ratio 5.23 \pm 0.27 ) pretreated groups (Fig. 4B). All animals pretreated with AAV9 .SUMO-1 retained cardiac function to the same degree as the mice injected with AAV9.SERCA2a. No statistically significant changes in echocardiographical parameters were observed in sham-operated groups (Table 3). The TAC operation significantly increased fibrosis in the AAV9.GFP control group compared with sham $(2.1 \% \pm 1.2 \%$ vs. $11.6 \% \pm 3.2 \%)$, but this increase was reduced in the TAC mice receiving either AAV9.SUMO-1 or AAV9.SERCA2a (Fig. 4C). As expected, the anti-hypertrophic effects of SUMO-1 prolong survival in animals with TAC-induced heart failure (Supplementary Fig. S3).

\section{SUMO-1 overexpression protects SERCA2a function from oxidative stress}

Oxidative stress plays an important role in the transition of cardiac hypertrophy to heart failure. SUMO-1 has also been demonstrated to play a role in stress responses, as its expression increases during hypoxia (59). We postulated that oxidative stress could inhibit SERCA2a function and SERCA2a SUMOylation status. To investigate the effects of SUMO-1 on oxidative stress-induced SERCA2a dysfunction, SERCA2a SUMOylation status, SERCA2a oxidation, and SERCA2a activity were determined in cardiomyocytes stimulated with $\mathrm{H}_{2} \mathrm{O}_{2}$. Isolated neonatal cardiomyocytes were infected with adenoviruses carrying $S U M O-1$ or $\beta$-gal genes for $24 \mathrm{~h}$ and then, the cells were exposed to $\mathrm{H}_{2} \mathrm{O}_{2}$ for $6 \mathrm{~h} . \mathrm{H}_{2} \mathrm{O}_{2}$ reduced SERCA2a SUMOylation as well as SERCA2a's ATPase activity (Fig. 5A), but endogenous levels of SERCA2a protein were not significantly changed. Furthermore, nitrosylated tyrosine residues of SERCA2a, which serve as a maker of oxidative post-translational modification, were significantly increased in $\mathrm{H}_{2} \mathrm{O}_{2}$-treated cardiomyocytes compared with no stimulation. However, pretreatment of SUMO-1 showed enhanced SERCA2a SUMOylation, reduced nitrosylation, and a $25 \%$ inhibited $\mathrm{H}_{2} \mathrm{O}_{2}$-mediated reduction of SERCA2a enzymatic activity (Fig. 5A).

To further define the mechanism underlying the enhanced antioxidant effects associated with SUMO-1, we next examined the effect of nicotinamide adenine dinucleotide phosphate oxidase 4 (Nox4) on SUMO-1 protein and SERCA2a SUMOylation during hypertrophy. Nox4 is a major enzyme that produces $\mathrm{H}_{2} \mathrm{O}_{2}$ in cardiomyocytes. Moreover, cardiac Nox4 expression level is increased in response to the PE and pressure overload is known to result in an increase oxidative stress and apoptosis. Interestingly, we observed that PE-induced upregulation of Nox4 expression was inhibited in SUMO-1 overexpressing cardiomyocytes (Supplementary Fig. S4). On the other hand, Western blot analyses showed that SUMO-1 expression, SERCA2a SUMOylation, and SERCA2a expression were preserved in the hearts isolated from cardiac-specific Nox4 knockout mice with TAC compared with wild-type mice with TAC (Fig. 5B). Furthermore, SERCA2a activity protected 

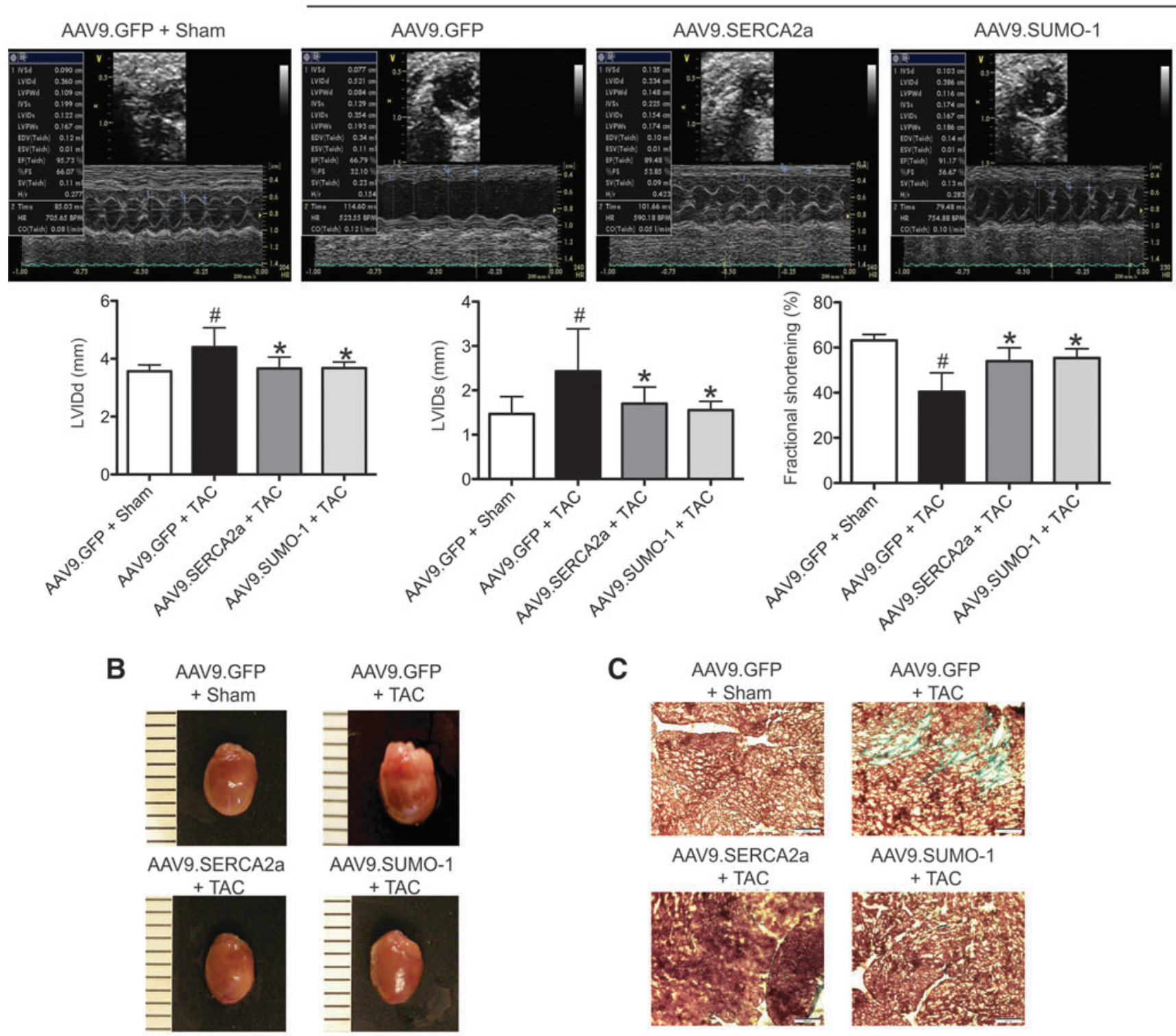

\section{C}
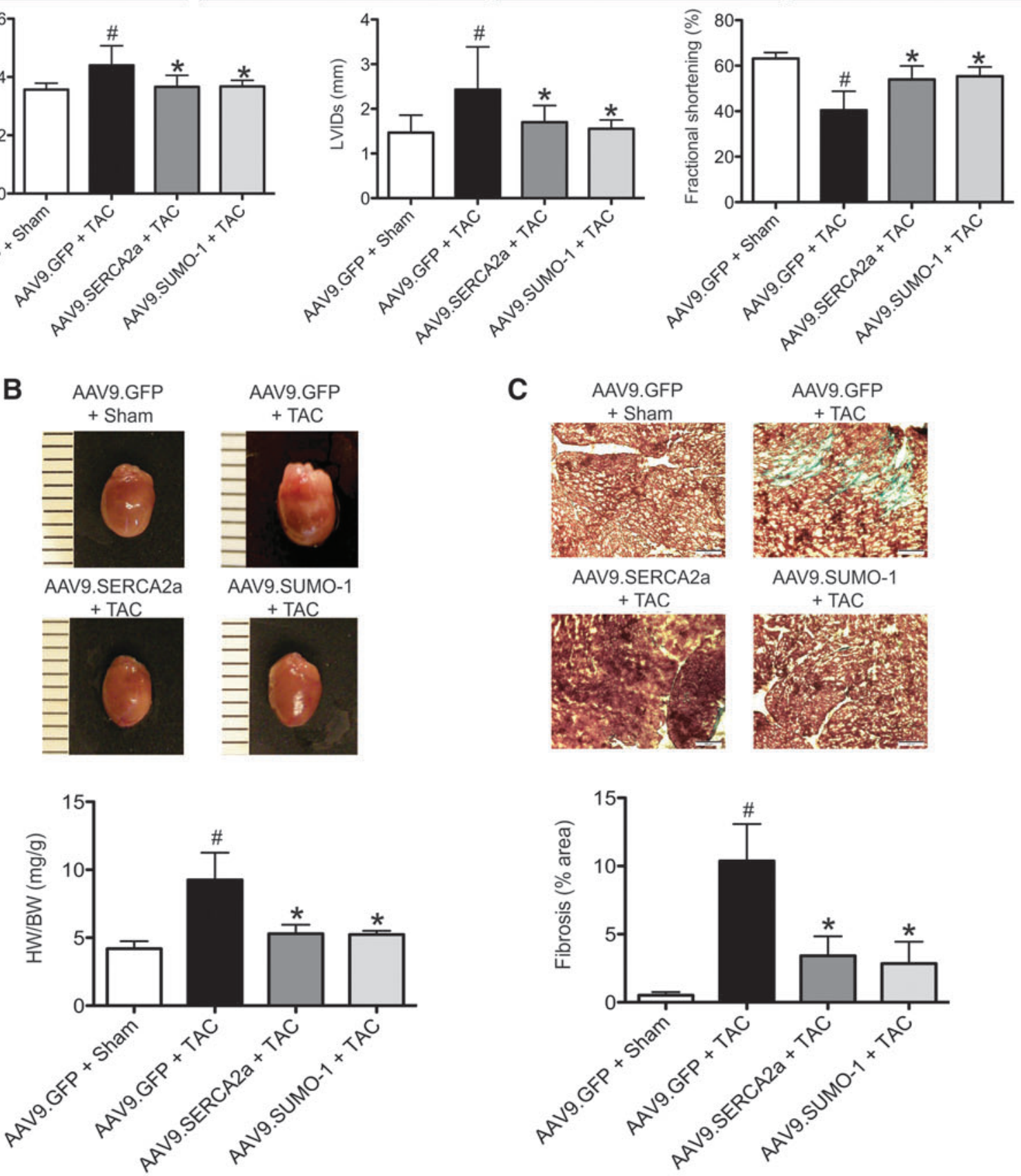

FIG. 4. SUMO-1 overexpression attenuates development of heart failure at 12 weeks after TAC mice. (A) Representative transthoracic M-mode echocardiogram for AAV9.GFP + TAC, AAV9.SERCA2a + TAC, and AAV9.SUMO-1 + TAC mice at 12 weeks of surgery is shown (top). LVIDd, LVIDs, and FS data for AAV9.GFP+TAC, AAV9.SERCA2a + TAC, and AAV9.SUMO-1 + TAC mice (bottom). (B) Gross heart image and ratios of heart weight to body weight are shown. (C) Representative Masson trichrome-stained sections and quantitative measurement. ${ }^{*} p<0.05$ versus GFP control with sham operation; * $p<0.05$ versus GFP control at 12 weeks of TAC. 
A

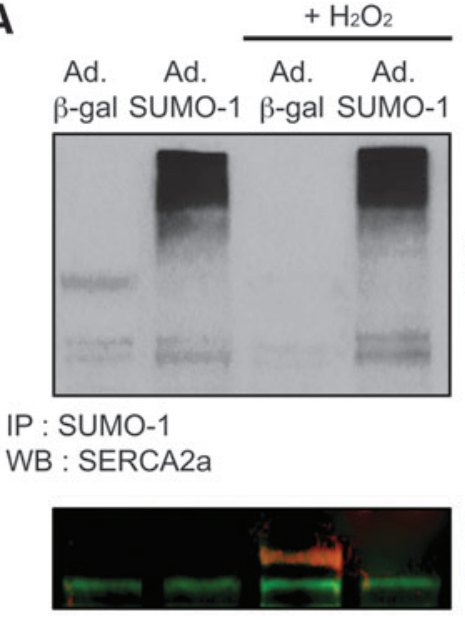

IP : SERCA2a

WB : Nitrityrosine (Red), SERCA2a (Green)

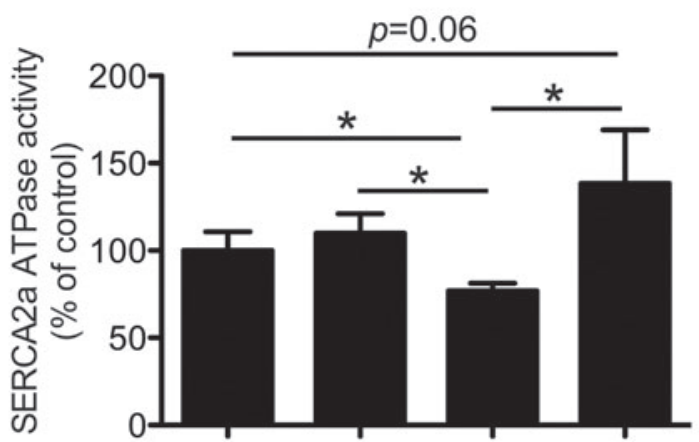

Nitrated SERCA2a

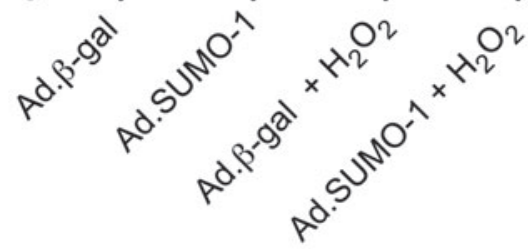

B

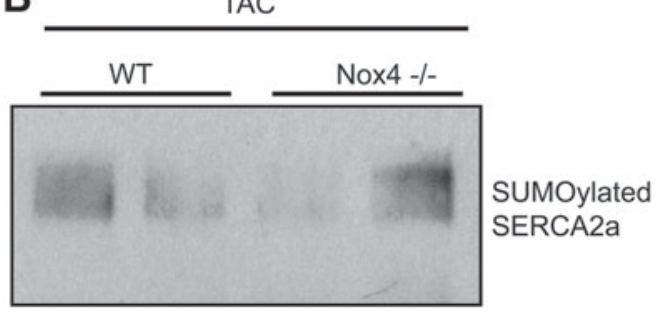

IP : SUMO-1

WB : SERCA2a

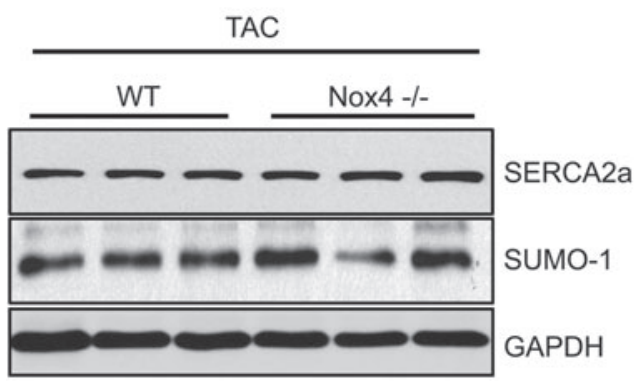

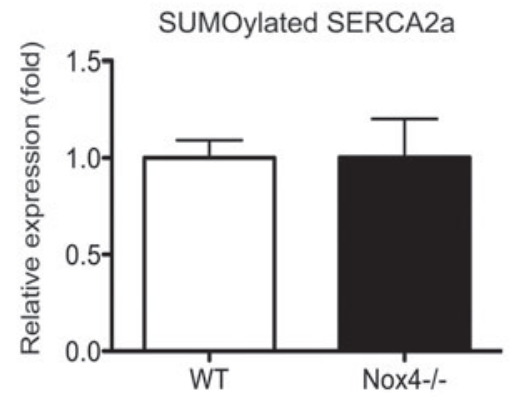
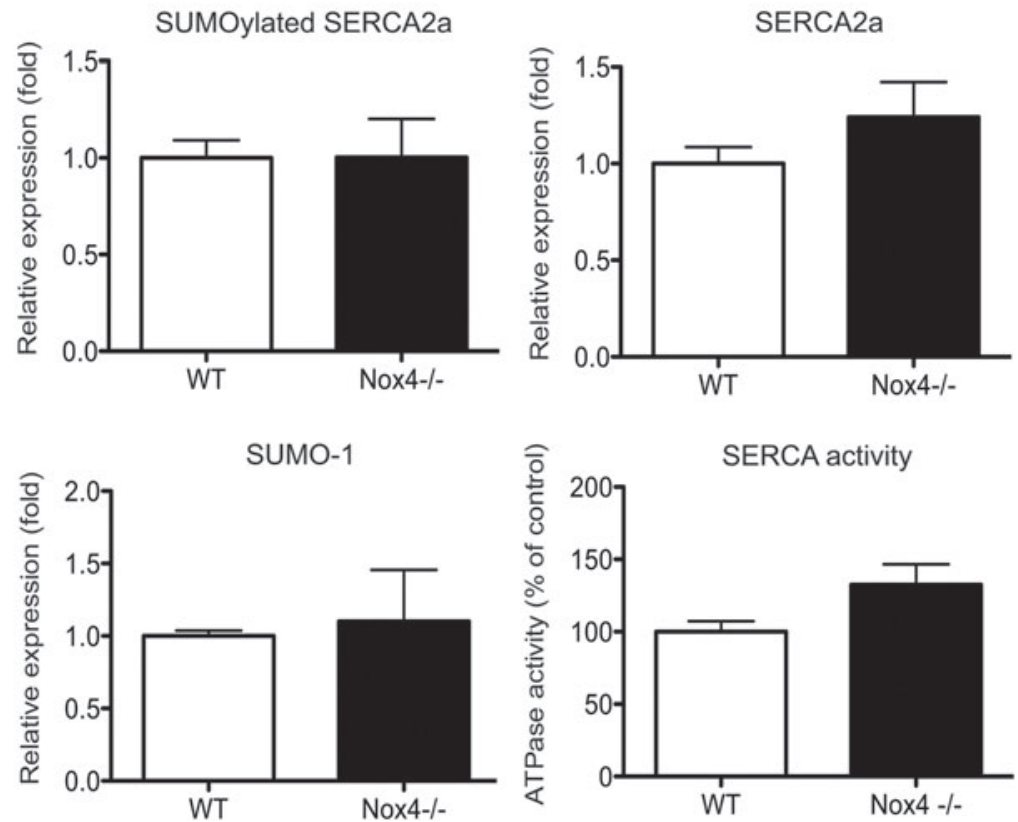

FIG. 5. SUMO-1 overexpression protects SERCA2a activity from oxidative stress. (A) Cardiomyocytes were infected with adenoviruses carrying SUMO- 1 or $\beta$-gal genes for $24 \mathrm{~h}$ and then, the cells were exposed to $0.5 \mu M$ of $_{2} \mathrm{H}_{2}$ for $6 \mathrm{~h}$. SERCA2a SUMOylation was determined by IP with anti-SUMO-1 followed by WB with anti-SERCA2a antibody. Tyrosine nitration of SERCA2a was measured by IP with anti-SERCA2a followed by WB with anti-3-nitrotyrosine antibody. The cell extracts were subjected to ATPase activity assay of SERCA2a. (B) Cardiac-specific Nox4 knockout mice were subjected to TAC operation for 4 weeks. The expression levels of SERCA2a, SUMO-1, and SUMOylated SERCA2a in cardiac tissues of Nox 4 knockout mice are shown. Data indicate the mean \pm SD of three independent experiments. ${ }^{*} p<0.05$ versus corresponding controls. Nox4, nicotinamide adenine dinucleotide phosphate oxidase 4. To see this illustration in color, the reader is referred to the web version of this article at www.liebertpub.com/ars

from TAC operation in Nox4 is deficient in mice (Fig. 5C). Taken together, these data suggest a protective role of SUMO1 in oxidative stress-induced SERCA2a dysfunction and Nox4-derived oxidative stress may influence SERCA2a SUMOylation and protein expression as well as its activity.

\section{SUMO-1 pretreatment reduces the TAC-induced increase in oxidative stress and reduction of SERCA2a level}

In an attempt to gain insights into the mechanisms by which overexpression of SUMO-1 confers resistance to ox- idative stress, we examined whether TAC induces generation of ROS. ROS levels in myocardial tissues were measured by dichlorofluorescein fluorescence (Fig. 6A). There was a 1.8 -fold increase in ROS production in TAC hearts preinjected with AAV9.GFP compared with sham control. However, SUMO-1 overexpression significantly prevented TAC-induced ROS generation. To further examine the extent of oxidative stress in the heart, the level of 4-hydroxynonenal (HNE)-modified protein was assessed in cardiac tissues using immunohistochemical staining with anti-4-HNE antibody. 4HNE-modified protein adducts in the hearts of TAC mice were 
significantly increased compared with sham-operated controls. However, TAC-induced HNE formation was significantly decreased in the hearts overexpressing SUMO-1 (Fig. 6B). Furthermore, TAC animals exhibited significant increases in the level of nitrotyrosine on SERCA2a and decreased SERCA2a SUMOylation. In contrast, SUMO-1 overexpressing hearts showed a decrease in SERCA2a oxidation and increases in the level of SUMOylated SERCA2a (Fig. 6C). SUMO-1 pretreatment also resulted in a strong restoration of SERCA2a protein levels at 12 weeks of TAC mice (Supplementary Fig. S5). These results point to the possibility that SUMO-1 overexpression contributes to diminished oxidative stress by reducing ROS levels.

\section{SUMO-1 overexpression contributes toward regulating redox signaling-mediated cardiac stress}

We next examined whether the anti-oxidant effect of SUMO-1 overexpression is correlated with redox-sensitive signaling molecules in pressure-overload-induced heart failure mice. Similar to the observation in vitro cardiomyocytes, TAC-induced activation of MAPK declined in SUMO-1 overexpressing hearts. TAC significantly elevated phosphorylation of ERK and JNK, and this activation subsequently affected increased phosphorylation of c-Jun (Fig. 6D). However, we did not observe a significant induction of MAPKs and c-Jun phosphorylation in TAC hearts that received AAV9.SUMO-1. We further tested the relationship between MAPK signaling and SUMO-1 in the pathogenic progression. In isolated neonatal cardiomyocytes, inhibition of the MEK/ ERK pathway by U0126 prevented PE-induced downregulation of SERCA2a expression; whereas the JNK inhibitor SP600125 had no such effect (Supplementary Fig. S6A). In the presence of PE, SUMO-1 expression was increased in the cells pretreated with JNK inhibitor (SP600125) compared with the cells without reagent (Supplementary Fig. S6B).

In mice preinjected with AAV9.GFP and subjected to 12 weeks of TAC, Nox4 protein level was significantly increased compared with sham-operated mice preinjected with AAV9.GFP (1.7-fold increase, $p<0.05)$. Interestingly, Nox4 level was greatly reduced in SUMO-1 overexpressing hearts with TAC. To further understand how the SUMO-1 overexpression leads to downregulation of Nox4, we checked the SUMOylation status of c-Jun, which is an important transcriptional regulator of Nox4 expression. SUMOylation of c-Jun was significantly increased in TAC mice pretreated with AAV9.SUMO-1 compared with any other groups, and this suggests an involvement of SUMO-1 in Nox regulation in vivo. In contrast, the level of mitochondrial anti-oxidant, MnSOD was decreased in the TAC mice injected with AAV9.GFP (38\% decrease) compared with sham control $(p<0.05)$. However, MnSOD expression was normalized in AAV9.SUMO-1 pretreated groups. Furthermore, SUMO-1 overexpression reduced the TAC-induced phosphorylation of PERK, a marker of endoplasmic reticulum (ER) stress (Fig. 6D). Almost molecular profiles were identical between SUMO-1 and SERCA2a pretreatment groups. These data indicate that pretreatment of SUMO-1 decreased oxidative stress and partially contributed to TAC-induced SERCA2a dysfunction and a decrease of LV function.

\section{Discussion}

SUMO belongs to a large family of ubiquitin (Ub)-related proteins. The overall sequence identity with $\mathrm{Ub}$ is small (around 18\%); however, the $C$-terminus of the protein, which confers most of its activity, is almost super-imposable to the equivalent region of $\mathrm{Ub}(3,50)$. Both SUMOylation and ubiquitination induce reversible covalent post-translational modification of lysine amino-acid residues in their substrate proteins, leading to changes in stability, activity and/or subcellular localization of the modified proteins they target (15, 18, 20, 21). Unlike ubiquitination, SUMOylation does not tag substrate proteins for degradation. In fact, SUMOylation and ubiquitination may even counteract one another by competing for the same lysine residues to define the fate of substrate proteins $(7,12,23,26)$. SUMOylation has been found to be highly relevant in signal transduction, particularly in response to cellular stress and disease $(57,61)$. We have previously reported the beneficial effects of SUMOylation on SERCA2a's biological properties using a murine model of heart failure (34). More recently, the positive effects of SUMO-1 gene transfer on SERCA2a and cardiac function were further confirmed in a porcine model of ischemia (63), suggesting a huge therapeutic potential of SUMO-1 for heart failure.

In this study, we found that SUMO-1 overexpression via gene transfer prevents the development of cardiac hypertrophy both in vitro and in vivo. In addition, we found that SUMO-1 gene transfer blocks the transition from compensated hypertrophy to heart failure. We also found that SUMO-1 abrogates the damage of ROS formation in the setting of hypertrophy and failure of SERCA2a.

FIG. 6. SUMO-1 pretreatment has positive effects at cellular and molecular levels. Mice were intravenously injected with AAV vectors encoding SUMO-1, SERCA2a, and GFP control and then subjected to either TAC or sham operation for 12 weeks. (A) Intracellular ROS level, primarily hydrogen peroxide, was measured by DCF fluorescent intensity by using cardiac tissue homogenates. (B) Myocardial levels of 4-HNE, a marker of cell damage, were determined by 4-HNE staining. Frozen sections from each hearts were imaged by confocal microscopy. (C) Western blots show SERCA2a SUMOyaltion status and SERCA2a oxidation. SUMOylated SERCA2a was immunoprecipitated using anti-SUMO-1 antibody, and the protein was blotted for SERCA2a with a SERCA2a-specific antibody. After immunoprecipitation with anti-SERCA2a antibody, the protein was separated by SDS-PAGE and Western blotted for nitrorytyrosine antibody. (D) Western blots show the effect of SUMO-1 pretreatment on redox signaling molecules. Phosphorylation of ERK, JNK, and c-Jun, as well as their downstream target was evaluated in cardiac tissues, indicating AAV vectors injected with TAC and sham control mice. SUMOylation status of c-Jun was determined in the same group of mice. Expression levels of Nox4, MnSOD, and PERK phosphorylation, ER stress marker. GAPDH was used as a loading control. Data are normalized to sham-operated control mice expressed with GFP. $* p<0.05$ versus corresponding controls. Representative blots from two to three independent experiments are shown. ${ }^{*} p<0.05$ versus GFP control at 12 weeks of TAC; ${ }^{\#} p<0.05$ versus GFP control with sham operation. DCF, dichlorofluorescein; ER, endoplasmic reticulum; 4-HNE, 4-hydroxy-2nonenal; ROS, reactive oxygen species; SDS-PAGE, sodium dodecyl sulfate-polyacrylamide gel electrophoresis. 


\section{A}
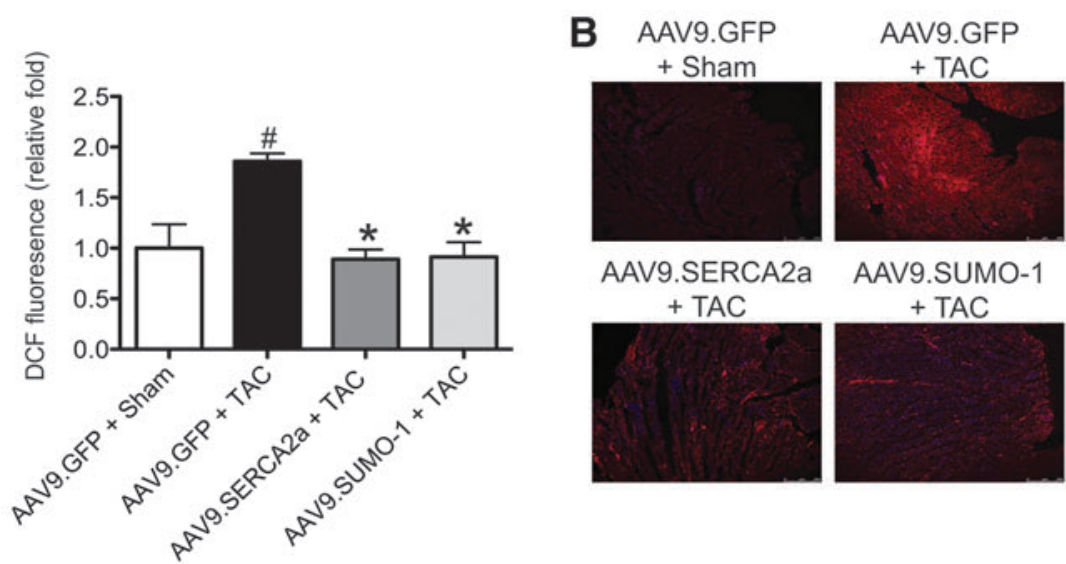

AAV9.SERCA2a

AAV9.SUMO-1

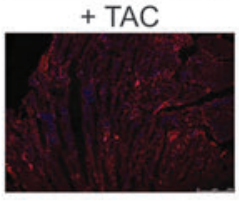

+ TAC
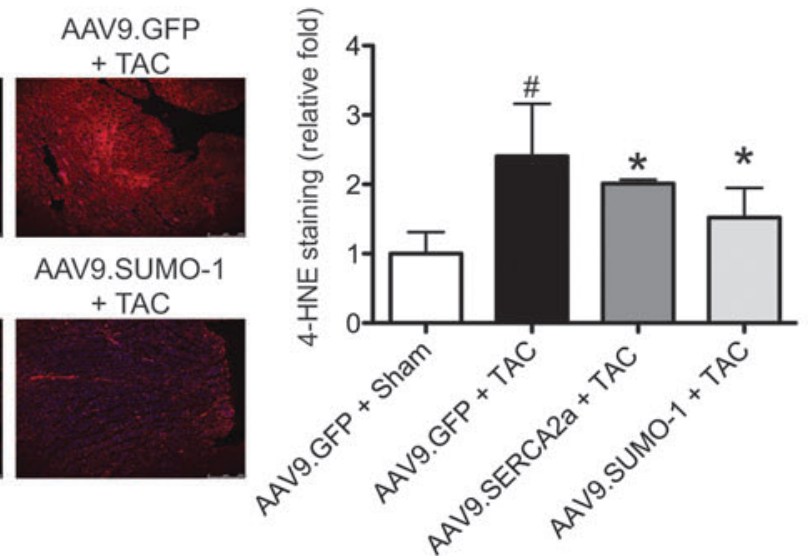

C

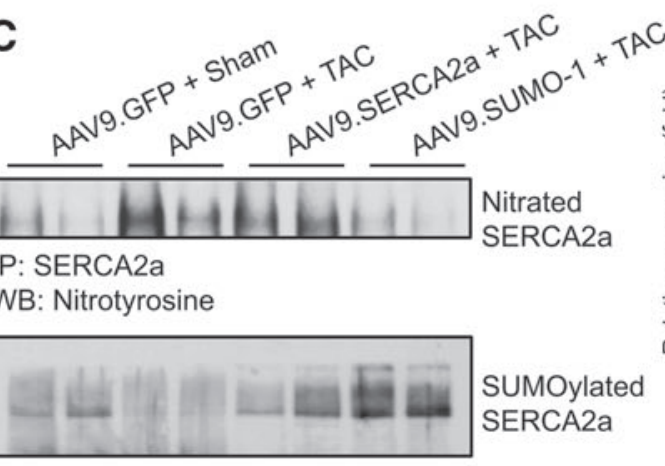

IP: SUMO-1

WB: SERCA2a

Nitrosylated SERCA2a

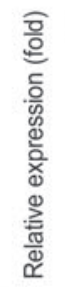

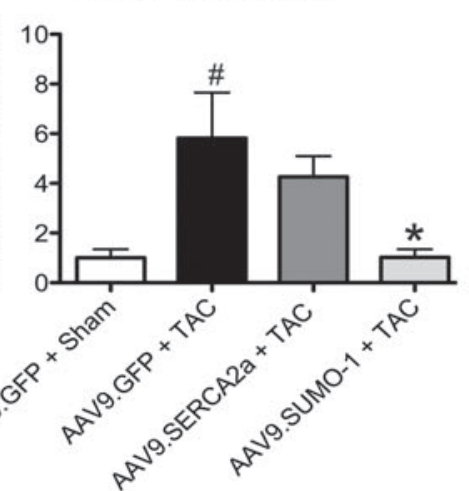

SUMOylated SERCA2a

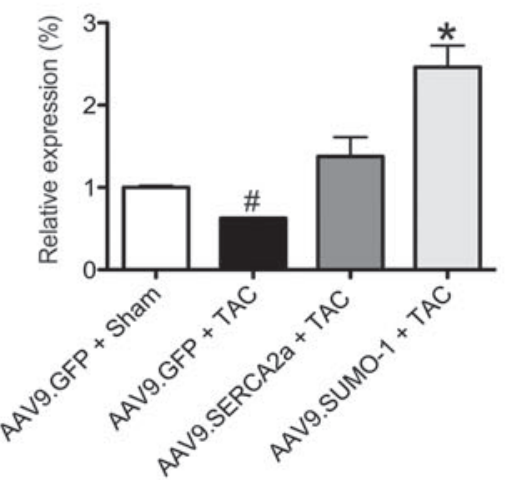

D

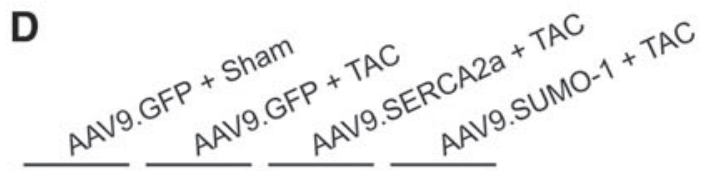

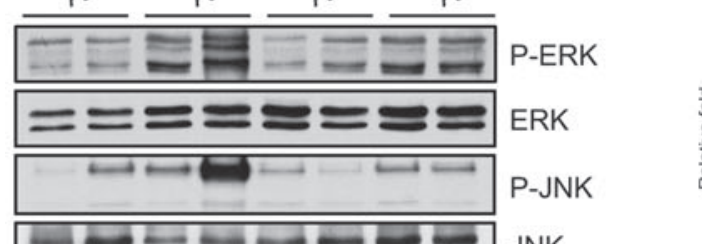
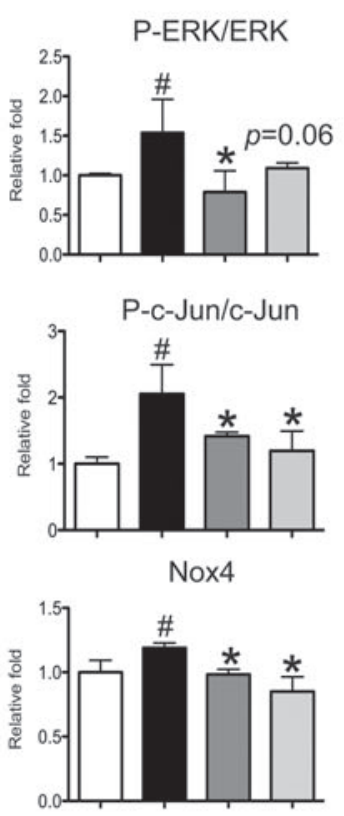
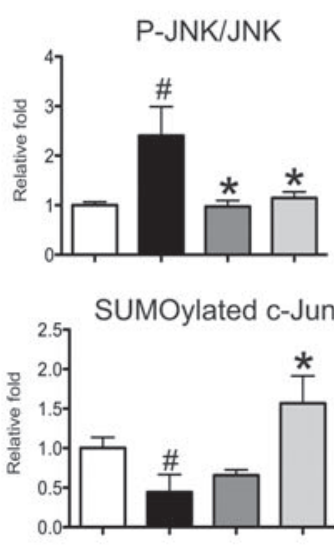

MnSOD
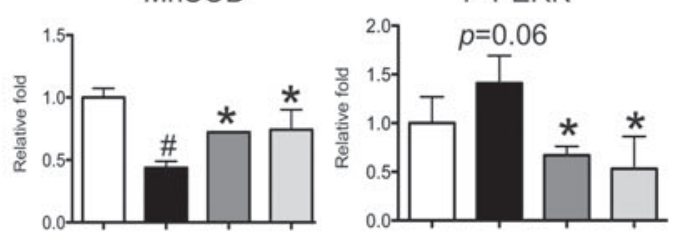

AAV9.GFP + Sham $\square$ AAV9.SERCA2a + TAC

AAV9.GFP + TAC $\square$ AAV9.SUMO-1 + TAC 
At the molecular level, we found that PE-induced MAPK activation was inhibited by either SUMO-1 or SERCA2a overexpression in cardiomyocytes. In TAC mice, SERCA2a overexpression seems more efficient in inhibiting MAPK activation (e.g., ERK) than SUMO-1 overexpression, suggesting that $\mathrm{Ca}^{2+}$ is an important upstream activator of MAPKs. Previously, it was shown that thapsigargin administration, an inhibitor of SERCA2a enzyme, induced ERK activity in human foreskin fibroblasts, underscoring the importance of SERCA2a in $\mathrm{Ca}^{2+}$-mediated MAPK activation (10). MAPK regulation by SUMO-1 seems to influence downstream signaling cascades. JNK activation was found to be in parallel with phosphorylation of c-Jun. Previous studies have reported that c-Jun activation has pro-hypertrophic and pro-apoptotic effects $(16,32,52)$. In this study, an increase in JNK-dependent phosphorylation of c-Jun in pressure-overloadinduced heart failure was significantly decreased by SUMO-1 overexpression. We tested the effects of MAPK inhibition on SUMO-1 and SERCA2a expression. PE-mediated MEK-ERK activation might induce SERCA2a downregulation, whereas JNK activation did not affect SERCA2a expression. In contrast, JNK activation during cardiac hypertrophy appears to contribute to SUMO-1 downregulation. These data strongly suggest that an association exists between MAPK and SUMO-1 in cardiomyocytes. The ERK and JNK are activated in response to hypertrophic stimuli in cultured cardiomyocytes $(4,11)$; however, the importance of this pathway and regulatory mechanisms in the cardiac hypertrophy remains controversial. Further studies, therefore, will be needed to investigate the detailed mechanisms underlying the regulation of each of the MAPKs vis a vis SUMO-1 and SERCA2a is to produce ROS; while all other major oxidases produce ROS as a secondary process either to catalyze another oxidative reaction or dysfunctional abnormal forms of enzymes generate ROS. The Nox family consists of seven members (Nox1-5, Duox1, and Duox2) and they are differentially expressed in various tissues. Nox1, Nox2, and Nox 5 mainly produce superoxide and Nox4 mainly produce hydrogen peroxide. Unlike other Nox family members, Nox4 activity is not controlled by binding regulatory proteins. The regulatory mechanism of Nox 4 in the heart, however, remains poorly understood. Previous studies have shown that Nox4 is elevated in pathological conditions (8, 38,42 ), and it directly impairs SERCA function (41). Induction of Nox4-derived ROS is known to coordinate the activity of ERK and JNK signaling by modulating ER stress signaling (65). In our study, we also observed an elevation of Nox4 expression when the MAPKs were activated in response to the hypertrophic stimuli such as $\mathrm{PE}$ in vitro and TAC in vivo. Recently, negative effects of SUMO-1 on Nox enzymes have been reported (53) by which Pandey et al. (53) showed that endogenous SUMO- 1 regulates ROS output and inhibits Nox activity, although they did not provide concrete evidence that Nox is a direct target of SUMO-1. These previous studies and our findings would suggest that there is a direct relationship between Nox4 and SUMO-1. It is interesting to note that Nox4 expression was decreased by SUMO-1 gene transfer. Increased SUMOylation of c-Jun by SUMO-1 overexpression may contribute to downregulation of Nox4 in pressure-overloaded hearts. A c-Jun/cfos-dependent transcriptional activation of Nox has been reported in previous studies (46). SUMOylation inhibits c-Jun/c-fos activity, which may turn off the Nox4 tran- scription. In SERCA2a overexpressing hearts, the level of Nox4 was also normalized. c-Jun SUMOylation seems to be increased (not statistically significant) compared with the control TAC hearts. Thus, c-Jun activation on phosphorylation by JNK, which is inhibited by either SUMO-1 or SERCA2a overexpression, may be involved in the regulation of Nox4. c-Jun activity is also closely associated with MnSOD transcripts and its activity. Endogenous c-Jun represses ROSreducing genes such as MnSOD and catalase via mitochondrial complexes and Nox enzyme (32).

At a cellular level, elevated levels of ROS impair cardiomyocyte function by damaging multiple channels and transporters $(24,43,69)$. ROS disrupts the structural integrity of ion channels via membrane lipid peroxidation $(17,37)$. ROS also decreases expression and activity of SERCA through posttranslational modifications $(1,31,58,60,68)$. Both tyrosine nitration and cystein oxidation of SERCA are known as important mechanisms that damage SERCA and lead to its decreased activity. Both these processes increase during aging. In this study, we observed the inhibitory effects of SUMO-1 pretreatment on SERCA2a oxidation, suggesting the potential role of SUMO-1 in the aging heart. Concomitant with these adverse effects on cardiomyocyte function, ROS stimulates a number of responses associated with the ventricular remodeling processes. These include ROS-mediated activation of matrix metalloproteinases to alter the architecture of the extracellular matrix, modulation of signal transduction pathways that initiate cardiomyocyte hypertrophy, and apoptosis or cell death $(9,35,39)$. Clearly, SUMO-1 can affect multiple critical proteins beyond SERCA2a that can lead to decreased oxidative stress.

Taken together, these observations suggest that one mechanism by which to halt deleterious ventricular remodeling and abnormal cardiomyocyte functional responses is by increasing SUMO-1. Concomitant with the protective effects of SUMO1 , we found a decrease in oxidative stress and interdependence between the Nox and SUMO pathways.

\section{Clinical implications}

More than 5.8 million Americans are suffering from heart failure, and there is an urgent unmet need for novel strategies to treat heart failure. In previous studies, we identified SUMO-1 as a new regulatory mechanism of SERCA2a showing that it enhanced its function and improved its protein stability and activity. We showed that in rodent and large animal models of heart failure, SUMO-1 gene transfer results in improved cardiac function and survival. In fact, co-expression of SUMO-1 and SERCA2a led to synergetic benefits. In this study, when we delivered SUMO-1 by gene transfer, we found that it protected SERCA2a from oxidative stresses that are prevalent in heart failure. We further found that SUMO-1 modulated important redox regulating molecules, which have been implicated in age-related heart diseases. Taken together, our results indicate that SUMO-1 may be a new therapeutic target not only in advanced heart failure but also during the early phases of transition from cardiac hypertrophy to failure.

\section{Materials and Methods}

\section{Animals and gene delivery}

All procedures were approved by the local Institutional Animal Care and Use Committee (IACUC). Male B6C3/F1 
mice were purchased from Charles River Laboratories. Six week-old male mice received AAV9 vectors carrying SUMO1, SERCA2a, and GFP via tail-vein injection. After 6 weeks of gene delivery, cardiac function was measured and randomized.

\section{Surgical interventions}

Mice underwent TAC using a supraclavicular construction model. TAC or sham surgery was performed at 6 weeks postgene delivery. Mice were anesthetized with intraperitoneal ketamine and placed on a ventilator. A 2- to 3-mm longitudinal cut was made in the proximal portion of the sternum, enabling visualization of the aortic arch. The transverse aortic arch was ligated between the innominate and left common carotid arteries with an overlaying 27-gauge needle. The needle was immediately removed, leaving a discrete region of constriction. The animal sham group underwent a similar procedure without ligation.

\section{Echocardiography}

Transthoracic echocardiography was performed using a Vivid 7000 GE machine equipped (GE Healthcare) with an H13L transducer (14 MHz). Two-dimensional and M-mode images were obtained in the short-axis view. The heart rate, left ventricular internal diameter in diastole (LVIDd), and left ventricular internal diameter in systole (LVIDs) were measured over the course of at least three repeated cardiac cycles. The EF and FS were then calculated.

\section{Cell culture and hypertrophic stimulation}

Primary cultures of cardiomyocytes from 2-day-old SpragueDawley rats were used. Briefly, ventricular tissue was enzymatically dissociated, and the resulting cardiomyocytes was isolated using Neonatal Cardiomyocyte isolation kit (Worthington Biochemical). Cells were plated onto collagen-coated culture dishes with a specific culture medium (DMEM supplemented with $10 \%$ fetal bovine serum and $2 \mathrm{~m} M$ L-glutamine; Life Technologies, Norwalk, CT). To induce hypertrophy, cardiomyocytes were cultured in serum-free medium for at least $24 \mathrm{~h}$ and then treated with $100 \mu M$ PE (Sigma-Aldrich).

\section{Quantitative RT-PCR}

Total RNA from NRVMs was isolated using RNeasy isolation kit (Qiagen) according to the manufacturer's instructions. cDNA was generated from total RNA using the Superscript II (Invitrogen). PCR was performed using an ABI Prism 7500 Real-Time PCR System. Results were expressed as target gene/18S ribosomal RNA. The following primers were used for qPCR:

ANF, sense 5'-CAGACCGATGAAGCGGGGGC-3', antisense 5'-CTTCGCAGGCTCCGAGGGC-3'; BNP, sense 5'-AG ACAAGAGAGAGCAGGACACC-3', antisense $5^{\prime}$-CTTGAA CTATGTGCCATCTTGG-3'; myh7, 5'-GAGGGCGGACATT GCCGAGT-3', 5'-AAGGCTCCAGGTCTCAGGGCTTC-3'; $18 \mathrm{~S}$, sense 5'-TCAAGAACGAAAGTCGGAGG-3', antisense 5'-GGACATCTAAGGGCATCAC-3'.

\section{Measurement of protein synthesis rate}

Isolated cardiomyocytes were cultured in serum-free DMEM medium for $12 \mathrm{~h}$. The cells then were stimulated with
PE for $12 \mathrm{~h}$ and incubated in the same medium containing $1.5 \mu \mathrm{Ci} / \mathrm{ml}\left[{ }^{3} \mathrm{H}\right]$ leucine (NEN Research Products) for an additional $20 \mathrm{~h}$. Total proteins were precipitated by the addition of $10 \%$ trichloroacetic acid for $45 \mathrm{~min}$ on ice, re-solubilized in $0.4 \mathrm{~N}$ sodium hydroxide for $1 \mathrm{~h}$ at $37^{\circ} \mathrm{C}$, and subjected to liquid scintillation counting.

\section{Molecular analysis}

The frozen tissues were crushed and homogenized in lysis buffer [50 $\mathrm{m} M$ Tris-Cl pH 8.0, $150 \mathrm{~m} M \mathrm{NaCl}, 0.1 \%$ Triton $\mathrm{X}-100,10 \mathrm{~m} M$ EDTA, complete protease inhibitor (one tablet per $10 \mathrm{ml}$; Roche), and a protein phosphatase inhibitor cocktail (Sigma-Aldrich)] using the MP homogenate system (FastPrep homogenizer). The insoluble portion was removed by centrifugation at $30,000 \mathrm{~g}$ for $20 \mathrm{~min}$. SERCA2a SUMOylation assay was performed as previously described (21). For in vivo SUMOylation assays, cardiac tissue extracts were immunoprecipitated with a SUMO-1 agarose resin (Santa Cruz Biotechnology) overnight at $4{ }^{\circ} \mathrm{C}$. The resins were washed thrice with cold lysis buffer. The immunocomplexes were then resolved by sodium dodecyl sulfatepolyacrylamide gel electrophoresis (SDS-PAGE), and Western blot analysis was performed using a SERCA2a-specific antibody. For SERCA2a oxidation analysis, SERCA2a was immunoprecipitated from cardiac tissue extracts with antiSERCA2a antibody. Nitrosylation of SERCA2a was then probed with the anti-nitrotyrosine antibody. For general Western blotting, cardiac proteins $(10-50 \mu \mathrm{g})$ were separated by SDS-PAGE, transferred to a nitrocellulose membrane (Bio-Rad), and probed with the following antibodies that were specific for SERCA2a (produced by 21st Century Biochemicals): glyceraldehyde 3-phosphate dehydrogenase (GAPDH) (Sigma-Aldrich), SUMO-1 (Santa Cruz Biotechnology), p-ERK, ERK, p-p38, p38, p-JNK, JNK, p-c-Jun, c-Jun, 3-nitrotyrosine (Cell Signaling), BNP (Santa Cruz Biotechnology), anti-rabbit-HRP, and anti-mouse-HRP secondary antibodies (Sigma-Aldrich). Blots were developed with Super Signal West Pico (Pierce). Protein band densities were quantified by using quantity ImageJ software (NIH). Inhibitors of ERK (U0126) and JNK (SP600125) were purchased from Calbiochem.

\section{Immunostaining}

Myocardial fibrosis was determined by Masson's trichrome staining (Sigma-Aldrich) of paraffin-embedded sections and semi-quantitatively rated. For 4-HNE staining, heart tissues were fixed and embedded. The sections were subjected to immunostaining with anti-HNE antibody (Alpha Diagnostics).

\section{Statistical analysis}

Results are presented as mean \pm SD. Data were analyzed using an unpaired $t$-test for comparisons between two means or a one-way ANOVA with the Bonferonni correction for comparisons between $>2$ means. A data comparison between the AAV9.SUMO-1 (or AAV9.SERCA2a) and AAV9 GFP groups was analyzed by using a post hoc Tukey test. $p<0.05$ was considered significant. Statistical analysis was performed using GraphPad Prism software (GraphPad Software, Inc.). 


\section{Acknowledgments}

This work is supported by NIH K99 HL116645, R01 HL093183, HL117505 01A1, HL088434, P20HL100396, an NHLBI Program of Excellence in Nanotechnology (PEN) Award, Contract No. HHSN268201000045C, and P50 HL112324.

\section{Author Disclosure Statement}

Dr Hajjar is the scientific cofounder of Celladon Corporation, which is developing AAV1.SERCA2a for the treatment of heart failure. No competing financial interests exist for the other authors.

\section{References}

1. Adachi T, Weisbrod RM, Pimentel DR, Ying J, Sharov VS, Schoneich C, and Cohen RA. S-Glutathiolation by peroxynitrite activates SERCA during arterial relaxation by nitric oxide. Nat Med 10: 1200-1207, 2004.

2. Amin JK, Xiao L, Pimental DR, Pagano PJ, Singh K, Sawyer DB, and Colucci WS. Reactive oxygen species mediate alpha-adrenergic receptor-stimulated hypertrophy in adult rat ventricular myocytes. J Mol Cell Cardiol 33: 131-139, 2001.

3. Bayer P, Arndt A, Metzger S, Mahajan R, Melchior F, Jaenicke R, and Becker J. Structure determination of the small ubiquitin-related modifier SUMO-1. J Mol Biol 280: 275-286, 1998.

4. Bogoyevitch MA, Glennon PE, Andersson MB, Clerk A, Lazou A, Marshall CJ, Parker PJ, and Sugden PH. Endothelin-1 and fibroblast growth factors stimulate the mitogen-activated protein kinase signaling cascade in cardiac myocytes. The potential role of the cascade in the integration of two signaling pathways leading to myocyte hypertrophy. J Biol Chem 269: 1110-1119, 1994.

5. Bossis G and Melchior F. Regulation of SUMOylation by reversible oxidation of SUMO conjugating enzymes. Mol Cell 21: 349-357, 2006.

6. Burgoyne JR, Mongue-Din H, Eaton P, and Shah AM. Redox signaling in cardiac physiology and pathology. Circ Res 111: 1091-1106, 2012.

7. Buschmann T, Fuchs SY, Lee CG, Pan ZQ, and Ronai Z. SUMO-1 modification of Mdm2 prevents its self-ubiquitination and increases Mdm2 ability to ubiquitinate p53. Cell 101: 753762,2000

8. Byrne JA, Grieve DJ, Bendall JK, Li JM, Gove C, Lambeth JD, Cave AC, and Shah AM. Contrasting roles of NADPH oxidase isoforms in pressure-overload versus angiotensin IIinduced cardiac hypertrophy. Circ Res 93: 802-805, 2003.

9. Cesselli D, Jakoniuk I, Barlucchi L, Beltrami AP, Hintze TH, Nadal-Ginard B, Kajstura J, Leri A, and Anversa P. Oxidative stress-mediated cardiac cell death is a major determinant of ventricular dysfunction and failure in dog dilated cardiomyopathy. Circ Res 89: 279-286, 2001.

10. Chao TS, Byron KL, Lee KM, Villereal M, and Rosner MR. Activation of MAP kinases by calcium-dependent and calciumindependent pathways. Stimulation by thapsigargin and epidermal growth factor. J Biol Chem 267: 19876-19883, 1992.

11. Clerk A, Bogoyevitch MA, Anderson MB, and Sugden PH. Differential activation of protein kinase $\mathrm{C}$ isoforms by endothelin-1 and phenylephrine and subsequent stimulation of p42 and p44 mitogen-activated protein kinases in ventricular myocytes cultured from neonatal rat hearts. $J$ Biol Chem 269: 32848-32857, 1994.
12. Desterro JM, Rodriguez MS, and Hay RT. SUMO-1 modification of IkappaBalpha inhibits NF-kappaB activation. Mol Cell 2: 233-239, 1998.

13. Dhalla AK, Hill MF, and Singal PK. Role of oxidative stress in transition of hypertrophy to heart failure. $\mathrm{J} \mathrm{Am}$ Coll Cardiol 28: 506-514, 1996.

14. Frey N and Olson EN. Cardiac hypertrophy: the good, the bad, and the ugly. Anпи Rev Physiol 65: 45-79, 2003.

15. Geiss-Friedlander R and Melchior F. Concepts in sumoylation: a decade on. Nat Rev Mol Cell Biol 8: 947-956, 2007.

16. Gillespie-Brown J, Fuller SJ, Bogoyevitch MA, Cowley S, and Sugden PH. The mitogen-activated protein kinase kinase MEK1 stimulates a pattern of gene expression typical of the hypertrophic phenotype in rat ventricular cardiomyocytes. J Biol Chem 270: 28092-28096, 1995.

17. Giordano FJ. Oxygen, oxidative stress, hypoxia, and heart failure. J Clin Invest 115: 500-508, 2005.

18. Haglund $\mathrm{K}$ and Dikic I. Ubiquitylation and cell signaling. EMBO J 24: 3353-3359, 2005.

19. Hajjar RJ, Zsebo K, Deckelbaum L, Thompson C, Rudy J, Yaroshinsky A, Ly H, Kawase Y, Wagner K, Borow K, Jaski B, London B, Greenberg B, Pauly DF, Patten R, Starling R, Mancini D, and Jessup M. Design of a phase 1/2 trial of intracoronary administration of AAV1/SERCA2a in patients with heart failure. J Card Fail 14: 355-367, 2008.

20. Hay RT. SUMO: a history of modification. Mol Cell 18: 1-12, 2005.

21. Hershko A and Ciechanover A. The ubiquitin system. Annu Rev Biochem 67: 425-479, 1998.

22. Hill MF and Singal PK. Right and left myocardial antioxidant responses during heart failure subsequent to myocardial infarction. Circulation 96: 2414-2420, 1997.

23. Hoege C, Pfander B, Moldovan GL, Pyrowolakis G, and Jentsch S. RAD6-dependent DNA repair is linked to modification of PCNA by ubiquitin and SUMO. Nature 419: 135-141, 2002.

24. Hool LC and Corry B. Redox control of calcium channels: from mechanisms to therapeutic opportunities. Antioxid Redox Signal 9: 409-435, 2007.

25. Hunter JJ and Chien KR. Signaling pathways for cardiac hypertrophy and failure. $N$ Engl J Med 341: 1276-1283, 1999.

26. Hunter T. The age of crosstalk: phosphorylation, ubiquitination, and beyond. Mol Cell 28: 730-738, 2007.

27. Ide T, Tsutsui H, Kinugawa S, Suematsu N, Hayashidani S, Ichikawa K, Utsumi H, Machida Y, Egashira K, and Takeshita A. Direct evidence for increased hydroxyl radicals originating from superoxide in the failing myocardium. Circ Res 86: 152-157, 2000.

28. Ito K, Yan X, Feng X, Manning WJ, Dillmann WH, and Lorell BH. Transgenic expression of sarcoplasmic reticulum $\mathrm{Ca}(2+)$ atpase modifies the transition from hypertrophy to early heart failure. Circ Res 89: 422-429, 2001.

29. Jessup M, Greenberg B, Mancini D, Cappola T, Pauly DF, Jaski B, Yaroshinsky A, Zsebo KM, Dittrich H, and Hajjar RJ. Calcium upregulation by percutaneous administration of gene therapy in cardiac disease I. Calcium upregulation by percutaneous administration of gene therapy in cardiac disease (CUPID): a phase 2 trial of intracoronary gene therapy of sarcoplasmic reticulum $\mathrm{Ca} 2+-\mathrm{ATPase}$ in patients with advanced heart failure. Circulation 124: 304-313, 2011.

30. Josephson RA, Silverman HS, Lakatta EG, Stern MD, and Zweier JL. Study of the mechanisms of hydrogen peroxide 
and hydroxyl free radical-induced cellular injury and calcium overload in cardiac myocytes. J Biol Chem 266: 2354-2361, 1991.

31. Kaplan P, Babusikova E, Lehotsky J, and Dobrota D. Free radical-induced protein modification and inhibition of $\mathrm{Ca} 2+-\mathrm{ATPase}$ of cardiac sarcoplasmic reticulum. Mol Cell Biochem 248: 41-47, 2003.

32. Katiyar S, Casimiro MC, Dettin L, Ju X, Wagner EF, Tanaka H, and Pestell RG. C-jun inhibits mammary apoptosis in vivo. Mol Biol Cell 21: 4264-4274, 2010.

33. Katz AM. Cardiomyopathy of overload. A major determinant of prognosis in congestive heart failure. $N$ Engl $\mathrm{J} \mathrm{Med}$ 322: 100-110, 1990.

34. Kho C, Lee A, Jeong D, Oh JG, Chaanine AH, Kizana E, Park WJ, and Hajjar RJ. SUMO1-dependent modulation of SERCA2a in heart failure. Nature 477: 601-605, 2011.

35. King MK, Coker ML, Goldberg A, McElmurray JH, 3rd, Gunasinghe HR, Mukherjee R, Zile MR, O'Neill TP, and Spinale FG. Selective matrix metalloproteinase inhibition with developing heart failure: effects on left ventricular function and structure. Circ Res 92: 177-185, 2003.

36. Kinugawa S, Tsutsui H, Hayashidani S, Ide T, Suematsu N, Satoh S, Utsumi H, and Takeshita A. Treatment with dimethylthiourea prevents left ventricular remodeling and failure after experimental myocardial infarction in mice: role of oxidative stress. Circ Res 87: 392-398, 2000.

37. Kourie JI. Interaction of reactive oxygen species with ion transport mechanisms. Am J Physiol 275: C1-C24, 1998.

38. Kuroda J, Ago T, Matsushima S, Zhai P, Schneider MD, and Sadoshima J. NADPH oxidase 4 (Nox4) is a major source of oxidative stress in the failing heart. Proc Natl Acad Sci U S A 107: 15565-15570, 2010.

39. Kwon SH, Pimentel DR, Remondino A, Sawyer DB, and Colucci WS. $\mathrm{H}(2) \mathrm{O}(2)$ regulates cardiac myocyte phenotype via concentration-dependent activation of distinct kinase pathways. J Mol Cell Cardiol 35: 615-621, 2003.

40. Lee YJ, Castri P, Bembry J, Maric D, Auh S, and Hallenbeck JM. SUMOylation participates in induction of ischemic tolerance. J Neurochem 109: 257-267, 2009.

41. Looi YH, Grieve DJ, Siva A, Walker SJ, Anilkumar N, Cave AC, Marber M, Monaghan MJ, and Shah AM. Involvement of Nox2 NADPH oxidase in adverse cardiac remodeling after myocardial infarction. Hypertension 51: 319-325, 2008.

42. Maalouf RM, Eid AA, Gorin YC, Block K, Escobar GP, Bailey S, and Abboud HE. Nox4-derived reactive oxygen species mediate cardiomyocyte injury in early type 1 diabetes. Am J Physiol Cell Physiol 302: C597-C604, 2012.

43. Madamanchi NR and Runge MS. Redox signaling in cardiovascular health and disease. Free Radic Biol Med 61C: 473-501, 2013.

44. Mak S, Lehotay DC, Yazdanpanah M, Azevedo ER, Liu PP, and Newton GE. Unsaturated aldehydes including 4-OH-nonenal are elevated in patients with congestive heart failure. J Card Fail 6: 108-114, 2000.

45. Mallat Z, Philip I, Lebret M, Chatel D, Maclouf J, and Tedgui A. Elevated levels of 8-iso-prostaglandin F2alpha in pericardial fluid of patients with heart failure: a potential role for in vivo oxidant stress in ventricular dilatation and progression to heart failure. Circulation 97: 1536-1539, 1998.

46. Manea A, Manea SA, Gafencu AV, Raicu M, and Simionescu M. AP-1-dependent transcriptional regulation of NADPH oxidase in human aortic smooth muscle cells: role of p22phox subunit. Arterioscler Thromb Vasc Biol 28: 878-885, 2008.

47. Manza LL, Codreanu SG, Stamer SL, Smith DL, Wells KS, Roberts RL, and Liebler DC. Global shifts in protein sumoylation in response to electrophile and oxidative stress. Chem Res Toxicol 17: 1706-1715, 2004.

48. Meguro T, Hong C, Asai K, Takagi G, McKinsey TA, Olson EN, and Vatner SF. Cyclosporine attenuates pressure-overload hypertrophy in mice while enhancing susceptibility to decompensation and heart failure. Circ Res 84: 735-740, 1999.

49. Miyamoto MI, del Monte F, Schmidt U, DiSalvo TS, Kang ZB, Matsui T, Guerrero JL, Gwathmey JK, Rosenzweig A, and Hajjar RJ. Adenoviral gene transfer of SERCA2a improves left-ventricular function in aortic-banded rats in transition to heart failure. Proc Natl Acad Sci U S A 97: 793-798, 2000.

50. Mossessova E and Lima CD. Ulp1-SUMO crystal structure and genetic analysis reveal conserved interactions and a regulatory element essential for cell growth in yeast. $\mathrm{Mol}$ Cell 5: 865-876, 2000.

51. Nakayama H, Otsu K, Yamaguchi O, Nishida K, Date MO, Hongo K, Kusakari Y, Toyofuku T, Hikoso S, Kashiwase K, Takeda T, Matsumura Y, Kurihara S, Hori M, and Tada M. Cardiac-specific overexpression of a high $\mathrm{Ca} 2+$ affinity mutant of SERCA2a attenuates in vivo pressure overload cardiac hypertrophy. FASEB J 17: 61-63, 2003.

52. Omura T, Yoshiyama M, Yoshida K, Nakamura Y, Kim S, Iwao H, Takeuchi K, and Yoshikawa J. Dominant negative mutant of c-Jun inhibits cardiomyocyte hypertrophy induced by endothelin 1 and phenylephrine. Hypertension 39: 81-86, 2002.

53. Pandey D, Chen F, Patel A, Wang CY, Dimitropoulou C, Patel VS, Rudic RD, Stepp DW, and Fulton DJ. SUMO1 negatively regulates reactive oxygen species production from NADPH oxidases. Arterioscler Thromb Vasc Biol 31: 1634-1642, 2011.

54. Pimentel DR, Amin JK, Xiao L, Miller T, Viereck J, Oliver-Krasinski J, Baliga R, Wang J, Siwik DA, Singh K, Pagano P, Colucci WS, and Sawyer DB. Reactive oxygen species mediate amplitude-dependent hypertrophic and apoptotic responses to mechanical stretch in cardiac myocytes. Circ Res 89: 453-460, 2001.

55. Qin F, Siwik DA, Lancel S, Zhang J, Kuster GM, Luptak I, Wang L, Tong X, Kang YJ, Cohen RA, and Colucci WS. Hydrogen peroxide-mediated SERCA cysteine 674 oxidation contributes to impaired cardiac myocyte relaxation in senescent mouse heart. J Am Heart Assoc 2: e000184, 2013.

56. Sam F, Kerstetter DL, Pimental DR, Mulukutla S, Tabaee A, Bristow MR, Colucci WS, and Sawyer DB. Increased reactive oxygen species production and functional alterations in antioxidant enzymes in human failing myocardium. J Card Fail 11: 473-480, 2005.

57. Sarge KD and Park-Sarge OK. Sumoylation and human disease pathogenesis. Trends Biochem Sci 34: 200-205, 2009.

58. Schoneich $\mathrm{C}$ and Sharov VS. Mass spectrometry of protein modifications by reactive oxygen and nitrogen species. Free Radic Biol Med 41: 1507-1520, 2006.

59. Shao R, Zhang FP, Tian F, Anders Friberg P, Wang X, Sjoland H, and Billig H. Increase of SUMO-1 expression in response to hypoxia: direct interaction with HIF-1alpha in adult mouse brain and heart in vivo. FEBS Lett 569: 293300, 2004. 
60. Sharov VS, Dremina ES, Galeva NA, Williams TD, and Schoneich C. Quantitative mapping of oxidation-sensitive cysteine residues in SERCA in vivo and in vitro by HPLCelectrospray-tandem MS: selective protein oxidation during biological aging. Biochem J 394: 605-615, 2006.

61. Skaug B and Chen ZJ. SUMO, ubiquitin, UBL proteins: implications for human diseases-fifth international conference. IDrugs 13: 224-227, 2010.

62. Srivastava S, Chandrasekar B, Bhatnagar A, and Prabhu SD. Lipid peroxidation-derived aldehydes and oxidative stress in the failing heart: role of aldose reductase. Am J Physiol Heart Circ Physiol 283: H2612-H2619, 2002.

63. Tilemann L, Lee A, Ishikawa K, Aguero J, Rapti K, SantosGallego C, Kohlbrenner E, Fish KM, Kho C, and Hajjar RJ. SUMO-1 gene transfer improves cardiac function in a largeanimal model of heart failure. Sci Transl Med 5: 211ra159, 2013.

64. van Berlo JH, Maillet M, and Molkentin JD. Signaling effectors underlying pathologic growth and remodeling of the heart. J Clin Invest 123: 37-45, 2013.

65. Wu RF, Ma Z, Liu Z, and Terada LS. Nox4-derived H2O2 mediates endoplasmic reticulum signaling through local Ras activation. Mol Cell Biol 30: 3553-3568, 2010.

66. Xiao L, Pimentel DR, Wang J, Singh K, Colucci WS, and Sawyer DB. Role of reactive oxygen species and NAD $(\mathrm{P}) \mathrm{H}$ oxidase in alpha(1)-adrenoceptor signaling in adult rat cardiac myocytes. Am J Physiol Cell Physiol 282: C926C934, 2002.

67. Xu KY, Zweier JL, and Becker LC. Hydroxyl radical inhibits sarcoplasmic reticulum $\mathrm{Ca}(2+)$-ATPase function by direct attack on the ATP binding site. Circ Res 80: 76-81, 1997.

68. Xu S, Ying J, Jiang B, Guo W, Adachi T, Sharov V, Lazar H, Menzoian J, Knyushko TV, Bigelow D, Schoneich C, and Cohen RA. Detection of sequence-specific tyrosine nitration of manganese SOD and SERCA in cardiovascular disease and aging. Am J Physiol Heart Circ Physiol 290: H2220-H2227, 2006.

69. Zima AV and Blatter LA. Redox regulation of cardiac calcium channels and transporters. Cardiovasc Res 71: 310-321, 2006.

70. Zsebo K, Yaroshinsky A, Rudy JJ, Wagner K, Greenberg B, Jessup M, and Hajjar RJ. Long-term effects of AAV1/ SERCA2a gene transfer in patients with severe heart failure: analysis of recurrent cardiovascular events and mortality. Circ Res 114: 101-108, 2014.

Address correspondence to: Dr. Changwon Kho Cardiovascular Research Center Icahn School of Medicine at Mount Sinai One Gustave Levy Place New York, NY 10029

E-mail: changwon.kho@mssm.edu

\author{
Dr. Roger J. Hajjar \\ Cardiovascular Research Center \\ Icahn School of Medicine at Mount Sinai \\ One Gustave Levy Place \\ New York, NY 10029
}

E-mail: roger.hajjar@mssm.edu

Date of first submission to ARS Central, May 6, 2014; date of acceptance, May 31, 2014.

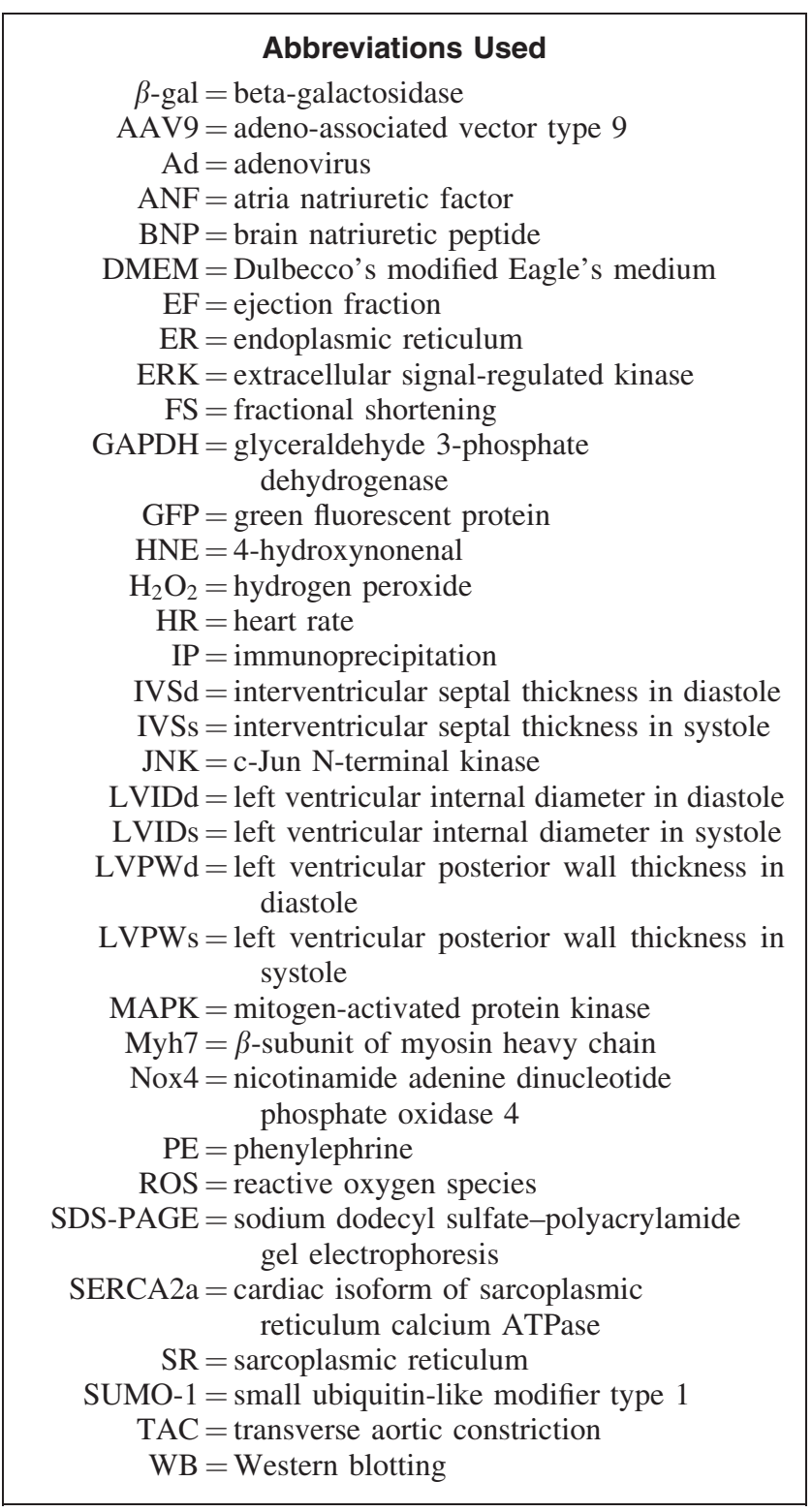

